Discrete Comput Geom 30:543-571 (2003)

DOI: $10.1007 / \mathrm{s} 00454-003-0789-4$

\title{
Common Transversals and Tangents to Two Lines and Two Quadrics in $\mathbb{P}^{3 *}$
}

\author{
Gábor Megyesi ${ }^{1}$, Frank Sottile $^{2}$, and Thorsten Theobald ${ }^{3}$ \\ ${ }^{1}$ Department of Mathematics, UMIST, \\ P.O. Box 88, Manchester, M60 1QD, England \\ gmegyesi@ma.umist.ac.uk \\ ${ }^{2}$ Department of Mathematics, University of Massachusetts, \\ Amherst, MA 01003, USA \\ sottile@math.umass.edu \\ ${ }^{3}$ Zentrum Mathematik, Technische Universität München, \\ Boltzmannstrasse 3, D-85747 Garching bei München, Germany \\ theobald@mathematik.tu-muenchen.de
}

\begin{abstract}
We solve the following geometric problem, which arises in several threedimensional applications in computational geometry: For which arrangements of two lines and two spheres in $\mathbb{R}^{3}$ are there infinitely many lines simultaneously transversal to the two lines and tangent to the two spheres?

We also treat a generalization of this problem to projective quadrics. Replacing the spheres in $\mathbf{R}^{3}$ by quadrics in projective space $\mathbb{P}^{3}$, and fixing the lines and one general quadric, we give the following complete geometric description of the set of (second) quadrics for which the two lines and two quadrics have infinitely many transversals and tangents: in the nine-dimensional projective space $\mathbb{P}^{9}$ of quadrics, this is a curve of degree 24 consisting of 12 plane conics, a remarkably reducible variety.
\end{abstract}

\section{Introduction}

In [14], one of us (Theobald) considered arrangements of $k$ lines and 4- $k$ spheres in $\mathbb{R}^{3}$ having infinitely many lines simultaneously transversal to the $k$ lines and tangent to the $4-k$ spheres. Since for generic configurations of $k$ lines and $4-k$ spheres there are only finitely many common transversals/tangents, the goal was to characterize the

* The research of the second author was supported in part by NSF Grant DMS-0070494. 
non-generic configurations where the discrete and combinatorial nature of the problem is lost. One case left open was that of two lines and two spheres. We solve that here.

A second purpose is to develop and present a variety of techniques from computational algebraic geometry for tackling problems of this kind. Since not all our readers are familiar with these techniques, we explain and document these techniques, with the goal of increasing their applicability. For that reason, we first deal with the more general problem where we replace the spheres in $\mathbb{R}^{3}$ by general quadratic surfaces (hereafter quadrics) in complex projective 3 -space $\mathbb{P}^{3}$. In order to study the geometry of this problem, we fix two lines and a quadric in general position, and describe the set of (second) quadrics for which there are infinitely many common transversals/tangents in terms of an algebraic curve. It turns out that this set is an algebraic curve of degree 24 in the space $\mathbb{P}^{9}$ of quadrics. Factoring the ideal of this curve shows that it is remarkably reducible:

Theorem 1. Fix two skew lines $\ell_{1}$ and $\ell_{2}$ and a quadric $Q$ in $\mathbb{P}^{3}$ that is neither tangent to either line nor contains any common transversal to the lines. The closure of the set of quadrics $Q^{\prime}$ for which there are infinitely many lines simultaneously transversal to $\ell_{1}$ and $\ell_{2}$ and tangent to both $Q$ and to $Q^{\prime}$ is a curve of degree 24 in the $\mathbb{P}^{9}$ of quadrics. This curve consists of 12 plane conics.

We prove this theorem by investigating the ideal defining the algebraic curve describing the set of (second) quadrics. Based on this, we prove the theorem with the aid of a computer calculation in the computer algebra system SINGULAR [4]. As explained in Section 3, the success of that computation depends crucially upon the preceding analysis of the curve. Quite interestingly, there are real lines $\ell_{1}$ and $\ell_{2}$ and real quadrics $Q$ such that all 12 components of the curve of second quadrics are real. In general, given real lines $\ell_{1}$ and $\ell_{2}$, and a real quadric $Q$, not all of the 12 components are defined over the real numbers.

While the beautiful and sophisticated geometry of our fundamental problem on lines and quadrics could be sufficient motivation to study this geometric problem, the original motivation came from algorithmic problems in computational geometry. As explained in [14], problems of this type occur in applications where one is looking for a line or ray interacting (in the sense of "intersecting" or in the sense of "not intersecting") with a given set of three-dimensional bodies, if the class of admissible bodies consists of polytopes and spheres (respectively quadrics). Concrete application classes of this type include visibility computations with moving viewpoints [15], controlling a laser beam in manufacturing [10], or the design of envelope data structures supporting ray shooting queries (i.e., seeking the first sphere, if any, met by a query ray) [1]. With regard to related treatments of the resulting algebraic-geometric core problems, we refer to [7], [8], and [13]. In these papers the question of arrangements of four (unit) spheres in $\mathbb{R}^{3}$ leading to an infinite number of common tangent lines is discussed from various viewpoints.

The present paper is structured as follows: In Section 1 we review the well-known Plücker coordinates from line geometry. In Section 2 we characterize the set of lines transversal to two skew lines and tangent to a quadric in terms of algebraic curves; we study and classify these $(2,2)$-curves. Then, in Section 3, we study the set of quadrics which (for prescribed lines $\ell_{1}$ and $\ell_{2}$ ) lead to most $(2,2)$-curves. This includes computer-algebraic calculations, based on which we establish the proof of Theorem 1. 
The Appendix to the paper contains annotated computer code used in the proof. In Section 4 we give some detailed examples illustrating the geometry described by Theorem 1 , and complete its proof. Finally, in Section 5, we solve the original question of spheres and give the complete characterization of configurations of two lines and two spheres having infinitely many lines transversal to the lines and tangent to the spheres. For a precise statement of that characterization see Theorems 16 and 20.

\section{Plücker Coordinates}

We review the well-known Plücker coordinates of lines in three-dimensional (complex) projective space $\mathbb{P}^{3}$. For general references, see [2], [6], and [11]. Let $x=\left(x_{0}, x_{1}, x_{2}, x_{3}\right)^{\mathrm{T}}$ and $y=\left(y_{0}, y_{1}, y_{2}, y_{3}\right)^{\mathrm{T}} \in \mathbb{P}^{3}$ be two points spanning a line $\ell$. Then $\ell$ can be represented (not uniquely) by the $4 \times 2$ matrix $L$ whose two columns are $x$ and $y$. The Plücker vector $p=\left(p_{01}, p_{02}, p_{03}, p_{12}, p_{13}, p_{23}\right)^{\mathrm{T}} \in \mathbb{P}^{5}$ of $\ell$ is the determinants of the $2 \times 2$ submatrices of $L$, that is, $p_{i j}:=x_{i} y_{j}-x_{j} y_{i}$. The set $\mathbb{G}_{1,3}$ of all lines in $\mathbb{P}^{3}$ is called the Grassmannian of lines in $\mathbb{P}^{3}$. The set of vectors in $\mathbb{P}^{5}$ satisfying the Plücker relation

$$
p_{01} p_{23}-p_{02} p_{13}+p_{03} p_{12}=0
$$

is in one-to-one correspondence with $\mathbb{G}_{1,3}$. See, for example Theorem 11 in section 8.6 of [2].

A line $\ell$ intersects a line $\ell^{\prime}$ in $\mathbb{P}^{3}$ if and only if their Plücker vectors $p$ and $p^{\prime}$ satisfy

$$
p_{01} p_{23}^{\prime}-p_{02} p_{13}^{\prime}+p_{03} p_{12}^{\prime}+p_{12} p_{03}^{\prime}-p_{13} p_{02}^{\prime}+p_{23} p_{01}^{\prime}=0
$$

Geometrically, this means that the set of lines intersecting a given line is described by a hyperplane section of the Plücker quadric (1.1) in $\mathbb{P}^{5}$.

In Plücker coordinates we also obtain a nice characterization (given in [13]) of the lines tangent to a given quadric in $\mathbb{P}^{3}$. (See [14] for an alternative deduction of that characterization.) We identify a quadric $x^{\mathrm{T}} Q x=0$ in $\mathbb{P}^{3}$ with its symmetric $4 \times 4$ representation matrix $Q$. Thus the sphere with center $\left(c_{1}, c_{2}, c_{3}\right)^{\mathrm{T}} \in \mathbb{R}^{3}$ and radius $r$ and described in $\mathbb{P}^{3}$ by $\left(x_{1}-c_{1} x_{0}\right)^{2}+\left(x_{2}-c_{2} x_{0}\right)^{2}+\left(x_{3}-c_{3} x_{0}\right)^{2}=r^{2} x_{0}^{2}$, is identified with the matrix

$$
\left(\begin{array}{cccc}
c_{1}^{2}+c_{2}^{2}+c_{3}^{2}-r^{2} & -c_{1}-c_{2} & -c_{3} \\
-c_{1} & 1 & 0 & 0 \\
-c_{2} & 0 & 1 & 0 \\
-c_{3} & 0 & 0 & 1
\end{array}\right)
$$

The quadric is smooth if its representation matrix has rank 4. To characterize the tangent lines, we use the second exterior power of matrices

$$
\wedge^{2}: \mathbb{C}^{m \times n} \rightarrow \mathbb{C}^{\left(\begin{array}{c}
m \\
2
\end{array}\right) \times\left(\begin{array}{l}
n \\
2
\end{array}\right)}
$$

(see p. 145 of [11], and [13]). Here $\mathbb{C}^{a \times b}$ is the set of $a \times b$ matrices with complex entries. The row and column indices of the resulting matrix are subsets of cardinality 2 
of $\{1, \ldots, m\}$ and $\{1, \ldots, n\}$, respectively. For $I=\left\{i_{1}, i_{2}\right\}$ with $1 \leq i_{1}<i_{2} \leq m$ and $J=\left\{j_{1}, j_{2}\right\}$ with $1 \leq j_{1}<j_{2} \leq n$,

$$
\left(\wedge^{2} A\right)_{I, J}:=A_{i_{1}, j_{1}} A_{i_{2}, j_{2}}-A_{i_{1}, j_{2}} A_{i_{2}, j_{1}} .
$$

Let $\ell$ be a line in $\mathbb{P}^{3}$ and let $L$ be a $4 \times 2$ matrix representing $\ell$. Interpreting the $6 \times 1$ matrix $\wedge^{2} L$ as a vector in $\mathbb{P}^{5}$, we observe that $\wedge^{2} L=p_{\ell}$, where $p_{\ell}$ is the Plücker vector of $\ell$.

Recall the following algebraic characterization of tangency: the restriction of the quadratic form to the line $\ell$ is singular, in that either it has a double root or it vanishes identically. When the quadric is smooth, this implies that the line is tangent to the quadric in the usual geometric sense.

Proposition 2 (Proposition 5.2 of [13]). A line $\ell \subset \mathbb{P}^{3}$ is tangent to a quadric $Q$ if and only if the Plücker vector $p_{\ell}$ of $\ell$ lies on the quadratic hypersurface in $\mathbb{P}^{5}$ defined by $\wedge^{2} Q$, if and only if

$$
p_{\ell}^{\mathrm{T}}\left(\wedge^{2} Q\right) p_{\ell}=0
$$

For a sphere with radius $r$ and center $\left(c_{1}, c_{2}, c_{3}\right)^{\mathrm{T}} \in \mathbb{R}^{3}$ the quadratic form $p_{\ell}^{\mathrm{T}}\left(\wedge^{2} Q\right) p_{\ell}$ is

$$
\left(\begin{array}{c}
p_{01} \\
p_{02} \\
p_{03} \\
p_{12} \\
p_{13} \\
p_{23}
\end{array}\right)^{\mathrm{T}}\left(\begin{array}{cccccc}
c_{2}^{2}+c_{3}^{2}-r^{2} & -c_{1} c_{2} & -c_{1} c_{3} & c_{2} & c_{3} & 0 \\
-c_{1} c_{2} & c_{1}^{2}+c_{3}^{2}-r^{2} & -c_{2} c_{3} & -c_{1} & 0 & c_{3} \\
-c_{1} c_{3} & -c_{2} c_{3} & c_{1}^{2}+c_{2}^{2}-r^{2} & 0 & -c_{1}-c_{2} \\
c_{2} & -c_{1} & 0 & 1 & 0 & 0 \\
c_{3} & 0 & -c_{1} & 0 & 1 & 0 \\
0 & c_{3} & -c_{2} & 0 & 0 & 1
\end{array}\right)\left(\begin{array}{l}
p_{01} \\
p_{02} \\
p_{03} \\
p_{12} \\
p_{13} \\
p_{23}
\end{array}\right)
$$

\section{Lines in $\mathbb{P}^{3}$ Meeting Two Lines and Tangent to a Quadric}

We work here over the ground field $\mathbb{C}$. First suppose that $\ell_{1}$ and $\ell_{2}$ are lines in $\mathbb{P}^{3}$ that meet at a point $p$ and thus span a plane $\Pi$. Then the common transversals to $\ell_{1}$ and $\ell_{2}$ either contain $p$ or they lie in the plane $\Pi$. This reduces any problem involving common transversals to $\ell_{1}$ and $\ell_{2}$ to a planar problem in $\mathbb{P}^{2}$ (or $\mathbb{R}^{2}$ ), and so we always assume that $\ell_{1}$ and $\ell_{2}$ are skew. Such lines have the form

$$
\begin{aligned}
& \ell_{1}=\left\{w a+x b:[w, x] \in \mathbb{P}^{1}\right\}, \\
& \ell_{2}=\left\{y c+z d:[y, z] \in \mathbb{P}^{1}\right\},
\end{aligned}
$$

where the points $a, b, c, d \in \mathbb{P}^{3}$ are affinely independent. We describe the set of lines meeting $\ell_{1}$ and $\ell_{2}$ that are also tangent to a smooth quadric $Q$. We refer to this set as the envelope of common transversals and tangents, or (when $\ell_{1}$ and $\ell_{2}$ are understood) simply as the envelope of $Q$.

The parametrization of (2.1) allows us to identify each of $\ell_{1}$ and $\ell_{2}$ with $\mathbb{P}^{1}$; the point $w a+x b \in \ell_{1}$ is identified with the parameter value $[w, x] \in \mathbb{P}^{1}$, and the same for $\ell_{2}$. We 
use these identifications throughout this section. In this way, any line meeting $\ell_{1}$ and $\ell_{2}$ can be identified with the pair $([w, x],[y, z]) \in \mathbb{P}^{1} \times \mathbb{P}^{1}$ corresponding to its intersections with $\ell_{1}$ and $\ell_{2}$. By (1.2), the Plücker coordinates $p_{\ell}=p_{\ell}(w, x, y, z)$ of the transversal $\ell$ passing through the points $w a+x b$ and $y c+z d$ are separately homogeneous of degree 1 in each set of variables $\{w, x\}$ and $\{y, z\}$, called bihomogeneous of bidegree $(1,1)$ (see, e.g., Section 8.5 of [2]).

By Proposition 2, the envelope of common transversals to $\ell_{1}$ and $\ell_{2}$ that are also tangent to $Q$ is given by the common transversals $\ell$ of $\ell_{1}$ and $\ell_{2}$ whose Plücker coordinates $p_{\ell}$ additionally satisfy $p_{\ell}^{\mathrm{T}}\left(\wedge^{2} Q\right) p_{\ell}=0$. This yields a homogeneous equation

$$
F(w, x, y, z):=p_{\ell}(w, x, y, z)^{\mathrm{T}}\left(\wedge^{2} Q\right) p_{\ell}(w, x, y, z)=0
$$

of degree 4 in the variables $w, x, y, z$. More precisely, $F$ has the form

$$
F(w, x, y, z)=\sum_{i, j=0}^{2} c_{i j} w^{i} x^{2-i} y^{j} z^{2-j}
$$

with coefficients $c_{i j}$, that is, $F$ is bihomogeneous with bidegree $(2,2)$. The zero set of a (non-zero) bihomogeneous polynomial defines an algebraic curve in $\mathbb{P}^{1} \times \mathbb{P}^{1}$ (see the treatment of projective elimination theory in Section 8.5 of [2]). In correspondence with its bidegree, the curve defined by $F$ is called a $(2,2)$-curve. The nine coefficients of this polynomial identify the set of $(2,2)$-curves with $\mathbb{P}^{8}$.

It is well known that the Cartesian product $\mathbb{P}^{1} \times \mathbb{P}^{1}$ is isomorphic to a smooth quadric surface in $\mathbb{P}^{3}$ [2, Proposition 10 in Section 8.6]. Thus the set of lines meeting $\ell_{1}$ and $\ell_{2}$ and tangent to the quadric $Q$ is described as the intersection of two quadrics in a projective 3-space. When it is smooth, this set is a genus 1 curve [5, Exercises I.7.2(d) and II.8.4(g)]. This set of lines cannot be parametrized by polynomials-only genus 0 curves (also called rational curves) admit such parametrizations (see, e.g., Corollary 2 on p.268 of [12]). This observation is the starting point for our study of common transversals and tangents.

Let $C$ be a $(2,2)$-curve in $\mathbb{P}^{1} \times \mathbb{P}^{1}$ defined by a bihomogeneous polynomial $F$ of bidegree 2 . The components of $C$ correspond to the irreducible factors of $F$, which are bihomogeneous of bidegree at most $(2,2)$. Thus any factors of $F$ must have bidegree 1 of $(2,2),(2,1),(1,1),(1,0)$, or $(0,1)$. (Since we are working over $\mathbb{C}$, a homogeneous quadratic of bidegree $(2,0)$ factors into two linear factors of bidegree $(1,0)$.) Recall (for example, [2]) that a point $\left(\left[w_{0}, x_{0}\right],\left[y_{0}, z_{0}\right]\right) \in C \subset \mathbb{P}^{1} \times \mathbb{P}^{1}$ is singular if the gradient $\nabla F$ vanishes at that point, $\nabla F\left(\left[w_{0}, x_{0}\right],\left[y_{0}, z_{0}\right]\right)=0$. The curve $C$ is smooth if it does not contain a singular point; otherwise $C$ is singular. We classify $(2,2)$-curves, up to change of coordinates on $\ell_{1} \times \ell_{2}$, and interchange of $\ell_{1}$ and $\ell_{2}$. Note that an $(a, b)$-curve and a $(c, d)$-curve meet if $a d+b c \neq 0$, and the intersection points are singular on the union of the two curves.

Lemma 3. Let $C$ be a $(2,2)$-curve on $\mathbb{P}^{1} \times \mathbb{P}^{1}$. Then, up to interchanging the factors of $\mathbb{P}^{1} \times \mathbb{P}^{1}, C$ is either

1. smooth and irreducible,

2. singular and irreducible, 
3. the union of a $(1,0)$-curve and an irreducible $(1,2)$-curve,

4. the union of two distinct irreducible $(1,1)$-curves,

5. a single irreducible $(1,1)$-curve, of multiplicity 2 ,

6. the union of one irreducible $(1,1)$-curve, one $(1,0)$-curve, and one $(0,1)$-curve,

7. the union of two distinct $(1,0)$-curves, and two distinct $(0,1)$-curves,

8. the union of two distinct $(1,0)$-curves, and one $(0,1)$-curve of multiplicity 2 ,

9. the union of one $(1,0)$-curve, and one $(0,1)$-curve, both of multiplicity 2.

In particular, when $C$ is smooth it is also irreducible.

When the polynomial $F$ has repeated factors, we are in cases (5), (8), or (9). We study the form $F$ when the quadric is reducible, that is, either when $Q$ has rank 1, so that it defines a double plane, or when $Q$ has rank 2, so that it defines the union of two planes.

Lemma 4. Suppose $Q$ is a reducible quadric.

(1) If $Q$ has rank 1 , then $\wedge^{2} Q=0$, and so the form $F$ in (2.2) is identically zero.

(2) Suppose $Q$ has rank 2, so that it defines the union of two planes meeting in a line $\ell$. If $\ell$ is one of $\ell_{1}$ or $\ell_{2}$, then the form $F$ in (2.2) is identically zero. Otherwise the form $F$ is the square of a $(1,1)$-form, and hence we are in cases (5) or (9) of Lemma 3.

Proof. The first statement is immediate. For the second, let $\ell^{\prime}$ be a line in $\mathbb{P}^{3}$ with Plücker coordinates $p_{\ell^{\prime}}$. From the algebraic characterization of tangency of Proposition 2, $p_{\ell^{\prime}}^{\mathrm{T}}\left(\wedge^{2} Q\right) p_{\ell^{\prime}}=0$ implies that the restriction of the quadratic form to $\ell^{\prime}$ either has a zero of multiplicity 2 , or it vanishes identically. In either case this implies that $\ell^{\prime}$ meets the line $\ell$ common to the two planes. Conversely, if $\ell^{\prime}$ meets the line $\ell$, then $p_{\ell^{\prime}}^{\mathrm{T}}\left(\wedge^{2} Q\right) p_{\ell^{\prime}}=0$.

Thus if $\ell$ equals one of $\ell_{1}$ or $\ell_{2}$, then $p_{\ell^{\prime}}^{\mathrm{T}}\left(\wedge^{2} Q\right) p_{\ell^{\prime}}=0$ for every common transversal $\ell^{\prime}$ to $\ell_{1}$ and $\ell_{2}$, and so the form $F$ is identically zero. Suppose that $\ell$ is distinct from both $\ell_{1}$ and $\ell_{2}$. We observed earlier that the set of lines transversal to $\ell_{1}$ and $\ell_{2}$ that also meet $\ell$ is defined by a $(1,1)$-form $G$. Since the $(2,2)$-form $F$ defines the same set as does the $(1,1)$-form $G$, we must have that $F=G^{2}$, up to a constant factor.

As above, let $C$ be the $(2,2)$-curve defined by the polynomial $F$. For a fixed point $[w, x]$, the restriction of the polynomial $F$ to $[w, x] \times \mathbb{P}^{1}$ is a homogeneous quadratic polynomial in $y, z$. A line passing through $[w, x] \in \ell_{1}$ and the point of $\ell_{2}$ corresponding to any zero of this restriction is tangent to $Q$. This construction gives all lines tangent to $Q$ that contain the point $[w, x]$. We call the zeros of this restriction the fiber over $[w, x]$ of the projection of $C$ to $\ell_{1}$.

We investigate these fibers. Consider the polynomial $F$ as a polynomial in the variables $y, z$ with coefficients polynomials in $w, x$. The resulting quadratic polynomial in $y, z$ has discriminant

$$
\left(\sum_{i=0}^{2} c_{i 1} w^{i} x^{2-i}\right)^{2}-4\left(\sum_{i=0}^{2} c_{i 0} w^{i} x^{2-i}\right)\left(\sum_{i=0}^{2} c_{i 2} w^{i} x^{2-i}\right) .
$$


Lemma 5. If this discriminant vanishes identically, then the polynomial $F$ has a repeated factor.

Proof. Let $\alpha, \beta, \gamma$ be the coefficients of $y^{2}, y z, z^{2}$ in the polynomial $F$, respectively. Then we have $\beta^{2}=4 \alpha \gamma$, as the discriminant vanishes. Since the ring of polynomials in $w, x$ is a unique factorization domain, either $\alpha$ differs from $\gamma$ by a constant factor, or else both $\alpha$ and $\gamma$ are squares. If $\alpha$ and $\gamma$ differ by a constant factor, then so do $\alpha$ and $\beta$. Writing $\beta=2 d \alpha$ for some $d \in \mathbb{C}$, we have

$$
F=\alpha y^{2}+2 d \alpha y z+d^{2} \alpha z^{2}=\alpha(y+d z)^{2} .
$$

If we have $\alpha=\delta^{2}$ and $\gamma=\sigma^{2}$ for some linear polynomials $\delta$ and $\sigma$, then

$$
F=\delta^{2} y \pm 2 \delta \sigma y z+\sigma^{2} z^{2}=(\delta y \pm \sigma z)^{2} .
$$

The fiber of $C$ over the point $[w, x]$ of $\ell_{1}$ consists of two distinct points exactly when the discriminant does not vanish at $[w, x]$. Call the points $[w, x]$ of $\ell_{1}$ where the discriminant vanishes (so that the fiber does not consist of two distinct points) a ramification point of the projection from $C$ to $\ell_{1}$. Suppose that $F$ does not have repeated factors so that the discriminant does not vanish identically. Since the discriminant (2.4) has degree 4 , there are at most four ramification points of $C$. If $C$ is irreducible, then these are the points where the fiber consists of a double point rather than two distinct points.

This discussion shows how we may parametrize the curve $C$, at least locally. Suppose that we have a point $[w, x] \in \mathbb{P}^{1}$ where the discriminant (2.4) does not vanish. Then we may solve for $[y, z]$ in the polynomial $F$ in terms of $[w, x]$. The different branches of the square root function give local parametrizations of the curve $C$.

\subsection{A Normal Form for Asymmetric Smooth (2, 2)-Curves}

Recall that for any distinct points $a_{1}, a_{2}, a_{3} \in \mathbb{P}^{1}$ and any distinct points $b_{1}, b_{2}, b_{3} \in \mathbb{P}^{1}$, there exists a projective linear transformation (given by a regular $2 \times 2$ matrix) which maps $a_{i}$ to $b_{i}, 1 \leq i \leq 3$ [2], [11].

Lemma 6. $A(2,2)$-curve $C$ is smooth if and only if its projection to $\ell_{1}$ has four distinct ramification points.

Proof. Suppose $C$ is a smooth (2,2)-curve. Changing coordinates on $\ell_{1}$ and $\ell_{2}$ by a projective linear transformation if necessary, we may assume that this projection to $\ell_{1}$ is ramified over $[w, x]=[1,0]$, and the double root of the fiber is at $[y, z]=[1,0]$. Restricting the polynomial $F(2.3)$ to the fiber over $[w, x]=[1,0]$ gives the equation

$$
c_{22} y^{2}+c_{21} y z+c_{20} z^{2}=0 .
$$

Since we assumed that this has a double root at $[y, z]=[1,0]$, we have $c_{21}=c_{22}=0$. 
Suppose now that the projection from $C$ to $\ell_{1}$ is ramified at fewer than four points. We may assume that $[w, x]=[1,0]$ is a double root of the discriminant (2.4), which implies that the coefficients of $w^{4}$ and $w^{3} x$ in (2.4) vanish. The previously derived condition $c_{21}=c_{22}=0$ implies that the coefficient of $w^{4}$ vanishes and the coefficient of $w^{3} x$ becomes $-4 c_{20} c_{12}$. If $c_{20}=0$, then every non-vanishing term of (2.3) depends on $x$; hence, $x$ divides $F$, and so $C$ is reducible, and hence not smooth. If $c_{12}=0$, then the gradient $\nabla F$ vanishes at the point $([1,0],[1,0])$, and so $C$ is not smooth.

Conversely, suppose that $C$ is not smooth. Assume that the point $([w, x],[y, z])=$ ([1, 0], [1, 0]) is a singular point of $C$. Then the projections of $C$ to $\ell_{1}$ and to $\ell_{2}$ are both ramified over [1,0]. We conclude as before that the coefficients $c_{21}, c_{22}$, and $c_{12}$ of the form $F$ (2.3) vanish. However, then the discriminant (2.4) is divisible by $x^{2}$, and so it has a double root. In particular, the projection of $C$ to $\ell_{1}$ has fewer than four ramification points.

Suppose that $C$ is a smooth $(2,2)$-curve. Then its projection to $\ell_{1}$ is ramified at four distinct points. We further assume that the double points in the ramified fibers project to at least three distinct points in $\ell_{2}$. We call such a smooth $(2,2)$-curve asymmetric. The choice of this terminology will become clear in Section 4. We will give a normal form for such asymmetric smooth curves.

Hence, we may assume that three of the ramification points are $[w, x]=[0,1],[1,0]$, and $[1,1]$, and the double points in these ramification fibers occur at $[y, z]=[0,1],[1,0]$, and $[1,1]$, respectively. As in the proof of Lemma 6 , the double point at $[y, z]=[1,0]$ in the fiber over $[w, x]=[1,0]$ implies that $c_{21}=c_{22}=0$. Similarly, the double point at $[y, z]=[0,1]$ in the fiber over $[w, x]=[0,1]$ implies that $c_{00}=c_{01}=0$. Thus the polynomial $F(2.3)$ becomes

$$
c_{20} w^{2} z^{2}+c_{10} w x z^{2}+c_{11} w x y z+c_{12} w x y^{2}+c_{02} x^{2} y^{2} .
$$

Restricting $F$ to the fiber of $[w, x]=[1,1]$ gives

$$
c_{10} z^{2}+c_{20} z^{2}+c_{11} y z+c_{02} y^{2}+c_{12} y^{2} .
$$

Since this has a double root at $[y, z]=[1,1]$, we must have

$$
-\frac{1}{2} c_{11}=c_{10}+c_{20}=c_{02}+c_{12} .
$$

Dehomogenizing (setting $c_{11}=-2$ ) and letting $c_{20}:=s$ and $c_{02}:=t$ for some $s, t \in \mathbb{C}$, we obtain the following theorem.

Theorem 7. After projective linear transformations in $\ell_{1}$ and $\ell_{2}$, an asymmetric smooth $(2,2)$-curve is the zero set of a polynomial

$$
s w^{2} z^{2}+(1-s) w x z^{2}-2 w x y z+(1-t) w x y^{2}+t x^{2} y^{2},
$$

for some $(s, t) \in \mathbb{C}^{2}$ satisfying

$$
s t(s-1)(t-1)(s-t) \neq 0 .
$$


We complete the proof of Theorem 7. The discriminant (2.4) of the polynomial (2.5) is

$$
4 w x(w-x)(s(t-1) w-t(s-1) x),
$$

which has roots at $[w, x]=[0,1],[1,0],[1,1]$, and $\alpha=[t(s-1), s(t-1)]$. Since we assumed that these are distinct, the fourth point $\alpha$ must differ from the first three, which implies that $(s, t)$ satisfies (2.6). The double point in the fiber over $\alpha$ occurs at $[y, z]=[s-1, t-1]$. This equals a double point in another ramification fiber only for values of the parameters not allowed by (2.6).

Remark 8. These calculations show that smooth $(2,2)$-curves exhibit the following dichotomy: either the double points in the ramification fibers project to four distinct points in $\ell_{2}$ or to two distinct points. They must project to at least two points, as there are at most two points in each fiber of the projection to $\ell_{2}$. We showed that if they project to at least three, then they project to four.

We compute the parameters $s$ and $t$ from the intrinsic geometry of the curve $C$. Recall the following definition of the cross ratio (see, for example Section 1.1.4 of [11]).

Definition 9. For four points $a_{1}, \ldots, a_{4} \in \mathbb{P}^{1}$ with $a_{i}=\left[\alpha_{i}, \beta_{i}\right]$, the cross ratio of $a_{1}, \ldots, a_{4}$ is the point of $\mathbb{P}^{1}$ defined by

$$
\left[\frac{\operatorname{det}\left(\begin{array}{cc}
\alpha_{1} & \alpha_{4} \\
\beta_{1} & \beta_{4}
\end{array}\right)}{\operatorname{det}\left(\begin{array}{cc}
\alpha_{1} & \alpha_{3} \\
\beta_{1} & \beta_{3}
\end{array}\right)}, \frac{\operatorname{det}\left(\begin{array}{cc}
\alpha_{2} & \alpha_{4} \\
\beta_{2} & \beta_{4}
\end{array}\right)}{\operatorname{det}\left(\begin{array}{ll}
\alpha_{2} & \alpha_{3} \\
\beta_{2} & \beta_{3}
\end{array}\right)}\right] .
$$

If the points are of the form $a_{i}=\left[1, \beta_{i}\right]$, this simplifies to

$$
\left[\frac{\beta_{4}-\beta_{1}}{\beta_{3}-\beta_{1}}, \frac{\beta_{4}-\beta_{2}}{\beta_{3}-\beta_{2}}\right] \text {. }
$$

The cross ratio of four points $a_{1}, a_{2}, a_{3}, a_{4} \in \mathbb{P}^{1}$ remains invariant under any projective linear transformation.

The projection of $C$ to $\ell_{1}$ is ramified over the points $[w, x]=[0,1],[1,0],[1,1]$ and $\alpha=[t(s-1), s(t-1)]$. The cross ratio of these four (ordered) ramification points is $[t(s-1), s(t-1)]$. Similarly, the cross ratio of the four (ordered) double points in the ramification fibers is $[s-1, t-1]$.

This computation of cross ratios allows us to compute the normal form of an asymmetric smooth $(2,2)$-curve. Namely, let $a_{1}, a_{2}, a_{3}$, and $a_{4}$ be the four ramification points of the projection of $C$ to $\ell_{1}$ and let $b_{1}, b_{2}, b_{3}$, and $b_{4}$ be the images in $\ell_{2}$ of the corresponding double points. Let $\gamma_{1}$ be the cross ratio of the four points $a_{1}, a_{2}, a_{3}$, and $a_{4}$ (this is well defined, as cross ratios are invariant under projective linear transformation). Similarly, let $\gamma_{2}$ be the cross ratio of the points $b_{1}, b_{2}, b_{3}$, and $b_{4}$. For four distinct points, 
the cross ratio is an element of $\mathbb{C} \backslash\{0,1\}$, so we express $\gamma_{1}, \gamma_{2}$ as complex numbers. The invariance of the cross ratios yields the conditions on $s$ and $t$ :

$$
\frac{s(t-1)}{t(s-1)}=\gamma_{1} \quad \text { and } \quad \frac{t-1}{s-1}=\gamma_{2}
$$

Again, since $\gamma_{1}, \gamma_{2} \in \mathbb{C} \backslash\{0,1\}$, these two equations have the unique solution

$$
s=\frac{\gamma_{1}\left(\gamma_{2}-1\right)}{\gamma_{2}\left(\gamma_{1}-1\right)} \quad \text { and } \quad t=\frac{\gamma_{2}-1}{\gamma_{1}-1} .
$$

Remark 10. We interpret the ramification of the $(2,2)$-curve $C$ of common tangents to a smooth quadric $Q$ geometrically and characterize when $C$ is smooth. Let $\ell_{1}$ and $\ell_{2}$ be skew lines and let $Q$ be a quadric whose tangent lines meeting $\ell_{1}$ and $\ell_{2}$ are described by the (2,2)-curve $C \subset \mathbb{P}^{1} \times \mathbb{P}^{1}$.

The fiber over $p \in \ell_{1}$ of the projection of $C$ to $\ell_{1}$ consists of the lines through $p$ meeting $\ell_{2}$ that are tangent to $Q$. These are the lines through $p$ lying in the plane $\Pi:=\overline{p, \ell_{2}}$ that are tangent to the conic $Q \cap \Pi$. Since $Q$ is smooth, either (a) $Q \cap \Pi$ is smooth or (b) $Q \cap \Pi$ consists of distinct lines $m, m^{\prime}$. In this second case, $\Pi$ is tangent to $Q$ at the point $q$ where the lines meet so that the tangents to $Q$ lying in $\Pi$ are the lines in $\Pi$ through $q$.

The curve $C$ is ramified over $p$ when the number of tangents to $Q \cap \Pi$ through $p$ is not two. That is, either (i) every line in $\Pi$ through $p$ is tangent to $Q$ or (ii) there is a single line in $\Pi$ through $p$ tangent to $Q$. In case (i), $Q$ is necessarily tangent to $\Pi$ at $p$, so that we are in (b) above with $p=q$. In this case, the fiber of $C$ over $p$ is a $(0,1)$-curve and so $C$ is reducible and hence not smooth. In case (ii), if (a) $Q \cap \Pi$ is smooth, then $p \in Q$ and the unique tangent is the tangent to this conic at $p$, and if (b) $Q \cap \Pi$ is singular, then $p \neq q$ and the unique tangent is the line $\overline{p, q}$.

We use this discussion and Lemma 6 to give a geometric characterization of when the $(2,2)$-curve is smooth.

Theorem 11. Let $\ell_{1}$ and $\ell_{2}$ be skew lines and let $Q$ be a smooth quadric in $\mathbb{P}^{3}$. Then the $(2,2)$-curve $C$ of common transversals to $\ell_{1}$ and $\ell_{2}$ that are tangent to $Q$ is smooth if and only if $Q$ is neither tangent to either line nor contains any common transversal to the lines.

Proof. Suppose that $Q$ is tangent to neither $\ell_{1}$ nor $\ell_{2}$ and that no common transversal to $\ell_{1}$ and $\ell_{2}$ lies in $Q$. Then $Q \cap \ell_{1}$ consists of two points $p, p^{\prime}$, each of which is a ramification point of the projection of $C$ to $\ell_{1}$. Also, exactly two planes $\Pi, \Pi^{\prime}$ containing $\ell_{2}$ are tangent to $Q$. Let $q, q^{\prime} \in \ell_{1}$ be the points of intersection of these tangent planes with $\ell_{1}$, which are also ramification points of the projection of $C$ to $\ell_{1}$. Suppose that $\left\{p, p^{\prime}\right\} \cap\left\{q, q^{\prime}\right\} \neq \emptyset$, say $p=q$. Then the plane $\Pi=\overline{p, \ell_{2}}$ is tangent to $Q$ and so it consists of two lines $m, m^{\prime}$. As $p \in Q, p$ lies on one of these lines, which is thus a common transversal to $\ell_{1}$ and $\ell_{2}$ lying in $Q$. However, this contradicts our assumptions, so we conclude that $\left\{p, p^{\prime}\right\} \cap\left\{q, q^{\prime}\right\}=\emptyset$. Thus the projection of $C$ to $\ell_{1}$ has four distinct ramification points and so by Lemma $6, C$ is smooth. 
Suppose now that $C$ is smooth. Then it is irreducible, and case (ii) of the discussion above cannot occur. By Lemma 6 the projection of $C$ to $\ell_{1}$ has four ramification points. These ramification points are those in $Q \cap \ell_{1}$ together with those points of $\ell_{1}$ lying in a tangent plane to $Q$ containing $\ell_{2}$. We do not have $\ell_{1} \subset Q$ or $\ell_{2} \subset Q$, as either implies that every point of $\ell_{1}$ is a ramification point (if $\ell_{2} \subset Q$, then every plane containing $\ell_{2}$ is tangent to $Q$ ). This implies that there are at most two points in $Q \cap \ell_{1}$ and at most two tangent plane to $Q$ containing $\ell_{2}$, and so there are exactly two of each type. However, then neither line is tangent to $Q$. Moreover, the points in $Q \cap \ell_{1}$ do not meet either tangent plane to $Q$ containing $\ell_{2}$, and the argument in the first paragraph shows this to be equivalent to the condition that $Q$ does not contain a common transversal to the two lines.

\section{Proof of Theorem 1}

We characterize the quadrics $Q$ which generate the same envelope of tangents as a given quadric. A symmetric $4 \times 4$ matrix has ten independent entries. This identifies the space of quadrics with $\mathbb{P}^{9}$. Central to our analysis is a map $\varphi$ defined for almost all quadrics $Q$. For a quadric $Q$ (considered as a point in $\mathbb{P}^{9}$ ) whose associated $(2,2)$-form $(2.2)$ is not identically zero, we let $\varphi(Q)$ be this $(2,2)$-form, considered as a point in $\mathbb{P}^{8}$. With this definition, we see that Theorem 1 is concerned with the fiber $\varphi^{-1}(C)$, where $C$ is the $(2,2)$-curve associated to a general quadric $Q$. Since the domain of $\varphi$ is nine-dimensional while its range is eight-dimensional, we expect each fiber to be one-dimensional.

We will show that every smooth $(2,2)$ curve arises as $\varphi(Q)$ for some quadric $Q$. This, together with Theorem 11, implies that Theorem 1 is a consequence of the following theorem.

Theorem 12. Let $C \in \mathbb{P}^{8}$ be a smooth $(2,2)$-curve. Then the closure $\overline{\varphi^{-1}(C)}$ in $\mathbb{P}^{9}$ of the fiber of $\varphi$ is a curve of degree 24 that is the union of 12 plane conics.

We prove Theorem 12 by computing the ideal $J$ of the fiber $\varphi^{-1}(C)$. Then we factor $J$ into several ideals, which corresponds to decomposing the curve of degree 24 into the union of several curves. Finally, we analyze the output of these computations by hand to prove the desired result.

Our initial formulation of the problem gives an ideal $I$ that not only defines the fiber of $\varphi$, but also the subset of $\mathbb{P}^{9}$ where $\varphi$ is not defined. We identify and remove this subset from $I$ in several costly auxiliary computations that are performed in the computer algebra system SINGULAR [4]. It is only after removing the excess components that we obtain the ideal $J$ of the fiber $\varphi^{-1}(C)$.

Since we want to analyze this decomposition for every smooth $(2,2)$-curve, we must treat the representation of $C$ as symbolic parameters. This leads to additional difficulties, which we circumvent. It is quite remarkable that the computer-algebraic calculation succeeds and that it is still possible to analyze its result.

In the following, we assume that $\ell_{1}$ is the $x$-axis. Furthermore, we may apply a projective linear transformation and assume without loss of generality that $\ell_{2}$ is the 
$y z$-line at infinity. Thus we have

$$
\begin{aligned}
& \ell_{1}=\left\{(w, x, 0,0)^{\mathrm{T}} \in \mathbb{P}^{3}:[w, x] \in \mathbb{P}^{1}\right\}, \\
& \ell_{2}=\left\{(0,0, y, z)^{\mathrm{T}} \in \mathbb{P}^{3}:[y, z] \in \mathbb{P}^{1}\right\} .
\end{aligned}
$$

Hence, in Plücker coordinates, the lines intersecting $\ell_{1}$ and $\ell_{2}$ are given by

$$
\left\{(0, w y, w z, x y, x z, 0)^{\mathrm{T}} \in \mathbb{P}^{5}:[w, x],[y, z] \in \mathbb{P}^{1}\right\} .
$$

By Proposition 2, the envelope of common transversals to $\ell_{1}$ and $\ell_{2}$ that are also tangent to $Q$ is given by those lines in (3.1) which additionally satisfy

$$
(0, w y, w z, x y, x z, 0)\left(\wedge^{2} Q\right)(0, w y, w z, x y, x z, 0)^{\mathrm{T}}=0 .
$$

A quadric $Q$ in $\mathbb{P}^{3}$ is given by the quadratic form associated to a symmetric $4 \times 4$ matrix

$$
Q:=\left(\begin{array}{llll}
a & b & c & d \\
b & e & f & g \\
c & f & h & k \\
d & g & k & l
\end{array}\right) .
$$

In a straightforward approach, the ideal $I$ of quadrics giving a general $(2,2)$-curve $C$ is obtained by first expanding the left-hand side of (3.2) into

$$
\begin{aligned}
\left(e l-g^{2}\right) x^{2} z^{2} & +2(b l-d g) w x z^{2}+\left(a l-d^{2}\right) w^{2} z^{2} \\
& +2(e k-g f) x^{2} y z+2(2 b k-c g-d f) w x y z \\
& +2(a k-d c) w^{2} y z \\
& +\left(e h-f^{2}\right) x^{2} y^{2}+2(b h-c f) w x y^{2}+\left(a h-c^{2}\right) w^{2} y^{2}
\end{aligned}
$$

We equate this $(2,2)$-form with the general $(2,2)$-form $(2.3)$, as points in $\mathbb{P}^{8}$. This is accomplished by requiring that they are proportional, or rather that the $2 \times 9$ matrix of their coefficients

$$
\left(\begin{array}{cccccc}
c_{00} & c_{10} & c_{20} & c_{01} & \cdots & c_{22} \\
e l-g^{2} & 2(b l-d g) & a l-d^{2} & 2(e k-g f) & \cdots & a h-c^{2}
\end{array}\right)
$$

has rank 1 . Thus the ideal $I$ is generated by the $\left(\begin{array}{l}9 \\ 2\end{array}\right)$ minors of this coefficient matrix.

With this formulation, the ideal $I$ will define the fiber $\varphi^{-1}(C)$ as well as additional, excess components that we wish to exclude. For example, the variety in $\mathbb{P}^{9}$ defined by the vanishing of the entries in the second row of this matrix will lie in the variety $I$, but these points are not those that we seek. Geometrically, these excess components are precisely where the map $\varphi$ is not defined. By Lemma 4, we can identify three of these excess components, those points of $\mathbb{P}^{9}$ corresponding to rank 1 quadrics, and those corresponding to rank 2 quadrics consisting of the union of two planes meeting in either $\ell_{1}$ or in $\ell_{2}$. The rank 1 quadrics have ideal $E_{1}$ generated by the entries of the matrix $\wedge^{2} Q$, 
the rank 2 quadrics whose planes meet in $\ell_{1}$ have ideal $E_{2}$ generated by $a, b, c, d, e, f, g$, and those whose plane meets in $\ell_{2}$ have ideal $E_{3}$ generated by $c, d, f, g, h, k, l$.

We remove these excess components from our ideal $I$ to obtain an ideal $J$ whose set of zeros contain the fiber $\varphi^{-1}(C)$. After factoring $J$ into its irreducible components, we will observe that $\varphi$ does not vanish identically on any component of $J$, completing the proof that $J$ is the ideal of $\varphi^{-1}(C)$, and also the proof of Theorem 12 .

Since $c_{00}, c_{10}, \ldots, c_{22}$ have to be treated as parameters, the computation should be carried out over the function field $\mathbb{Q}\left(c_{00}, c_{10}, \ldots, c_{22}\right)$. That computation is infeasible. Even the initial computation of a Gröbner basis for the ideal $I$ (a necessary prerequisite) did not terminate in 2 days. In contrast, the computation we finally describe terminates in 7 minutes on the same computer. This is because the original computation in $\mathbb{Q}\left(c_{00}, c_{10}, \ldots, c_{22}\right)[a, b, \ldots, l]$ involved too many parameters.

We instead use the two-parameter normal form $(2.5)$ for asymmetric smooth $(2,2)$ curves. This will prove Theorem 12 in the case when $C$ is an asymmetric smooth $(2,2)$ curve. We treat the remaining cases of symmetric smooth $(2,2)$-curves in Section 4 . As described in Section 2.1, by changing the coordinates on $\ell_{1}$ and $\ell_{2}$, every asymmetric smooth $(2,2)$-curve can be transformed into one defined by a polynomial in the family (2.5). Equating the (2, 2)-form (3.4) with the form (2.5) gives the ideal $I$ generated by the following polynomials:

$$
e l-g^{2}, e k-g f, a k-d c, a h-c^{2},
$$

and the ten $2 \times 2$ minors of the coefficient matrix:

$$
M:=\left(\begin{array}{ccccc}
s & 1-s & -2 & 1-t & t \\
a l-d^{2} & 2(b l-d g) & 2(2 b k-c g-d f) & 2(b h-c f) & e h-f^{2}
\end{array}\right) .
$$

This ideal $I$ defines the same three excess components as before, and we must remove them to obtain the desired ideal $J$. Although the ideal $I$ should be treated in the ring $S:=\mathbb{Q}(s, t)[a, b, c, d, e, f, g, h, k, l]$, the necessary calculations are infeasible even in this ring, and we instead work in subring $R:=\mathbb{Q}[a, b, c, d, e, f, g, h, k, l][s, t]$. In the ring $R$ the ideal $I$ is homogeneous in the set of variables $a, b, \ldots, l$, thus defining a subvariety of $\mathbb{P}^{9} \times \mathbb{C}^{2}$. The ideals $E_{1}, E_{2}$, and $E_{3}$ describing the excess components satisfy $E_{j} \supset I, 1 \leq j \leq 3$.

A SINGULAR computation shows that $I$ is a five-dimensional subvariety of $\mathbb{P}^{9} \times \mathbb{C}^{2}$ (see the Appendix for details). Moreover, the dimensions of the three excess components are 5,4 , and 4 , respectively. In fact, it is quite easy to see that $\operatorname{dim} E_{2}=\operatorname{dim} E_{3}=4$ as both ideals are defined by seven independent linear equations.

We are faced with a geometric situation of the following form. We have an ideal $I$ whose variety contains an excess component defined by an ideal $E$ and we want to compute the ideal of the difference

$$
\mathcal{V}(I)-\mathcal{V}(E)
$$

here, $\mathcal{V}(K)$ is the variety of an ideal $K$. Computational algebraic geometry gives us an effective method to accomplish this, namely saturation. The elementary notion is that of 
the ideal quotient $(I: E)$, which is defined by

$$
(I: E):=\{f \in R \mid f g \in I \text { for all } g \in E\} .
$$

Then the saturation of $I$ with respect to $E$ is

$$
\left(I: E^{\infty}\right):=\bigcup_{n=1}^{\infty}\left(I: E^{n}\right)
$$

The least number $n$ such that $\left(I: E^{\infty}\right)=\left(I: E^{n}\right)$ is called the saturation exponent.

Proposition 13 ([2, Section 4.4], [3, Section 15.10], or the reference manual for SINGULAR). Over an algebraically closed field,

$$
\mathcal{V}\left(I: E^{\infty}\right)=\overline{\mathcal{V}(I)-\mathcal{V}(E)}
$$

A Singular computation shows that the saturation exponent of the first excess ideal $E_{1}$ in $I$ is 1 , and so the ideal quotient suffices to remove the excess component $\mathcal{V}\left(E_{1}\right)$ from $\mathcal{V}(I)$. Set $I^{\prime}:=\left(I: E_{1}\right)$, an ideal of dimension 4 . The excess ideals $E_{2}$ and $E_{3}$ each have saturation exponent 4 in $I_{1}$, and so we saturate $I^{\prime}$ with respect to each to obtain an ideal $J:=\left(\left(I^{\prime}: E_{2}^{\infty}\right): E_{3}^{\infty}\right)$, which has dimension 3 in $\mathbb{P}^{9} \times \mathbb{C}^{2}$.

To study the components of $\mathcal{V}(J)$, we first apply the factorization Gröbner basis algorithm to $J$, as implemented in the SINGULAR command facsta (see [9] or the reference manual of SINGULAR). This algorithm takes two arguments, an ideal $I$ and a list $L=f_{1}, \ldots, f_{n}$ of polynomials. It proceeds as in the usual Buchberger algorithm to compute a Gröbner basis for $I$, except that whenever it computes a Gröbner basis element $G$ that it can factor, it splits the calculation into subcalculations, one for each factor of $G$ that is not in the list $L$, adding that factor to the Gröbner basis for the corresponding subcalculation. The output of facsta is a list $I_{1}, I_{2}, \ldots, I_{m}$ of ideals with the property that

$$
\overline{\bigcup_{j=1}^{m} \mathcal{V}\left(I_{j}\right)-\mathcal{V}\left(f_{1} \cdots f_{n}\right)}=\overline{\mathcal{V}(I)-\mathcal{V}\left(f_{1} \cdots f_{n}\right)} .
$$

Thus, the zero set of $I$ coincides with the union of zero sets of the factors $I_{j}$, in the region where none of the polynomials in the list $L$ vanish. In terms of saturation, this is

$$
\operatorname{rad}\left(I_{1} \cdots I_{m}:\left(f_{1} f_{2} \cdots f_{n}\right)^{\infty}\right)=\operatorname{rad}\left(I:\left(f_{1} f_{2} \cdots f_{n}\right)^{\infty}\right),
$$

where $\operatorname{rad}(K)$ denotes the radical of an ideal $K$. Some of the ideals $I_{j}$ may be spurious in that $\mathcal{V}\left(I_{j}\right)$ is already contained in the union of the other $\mathcal{V}\left(I_{i}\right)$.

We run facstd on the ideal $J$ with the list of polynomials $s, t, s-1, t-1$, and $s-t$, and obtain seven components $J_{0}, J_{1}, \ldots, J_{6}$. The components $J_{1}, \cdots, J_{6}$ each have dimension 3, while the component $J_{0}$ has dimension 2. Since $\mathcal{V}\left(J_{0}\right)$ is contained in the union of the $\mathcal{V}\left(J_{1}\right), \cdots, \mathcal{V}\left(J_{6}\right)$, it is spurious and so we disregard it.

We now, finally, change from the base ring $R$ to the base ring $S$, and compute with the parameters $s, t$. There, $J$ defines an ideal of dimension 1 and degree 24 in the ninedimensional projective space over the field $\mathbb{Q}(s, t)$. As we remarked before, we have that 
$\mathcal{V}(J) \supset \varphi^{-1}(C)$. The factorization of $J$ into $J_{1}, \ldots, J_{6}$ remains valid over $S$. The reason we did not compute the factorization over $S$ is that facsta and the saturations were infeasible over $S$, and the standard arguments from computational algebraic geometry we have given show that it suffices to compute without parameters, as long as care is taken when interpreting the output.

Each of the factors $J_{i}$ has dimension 1 and degree 4. Moreover, each ideal contains a homogeneous quadratic polynomial in the variables $k, l$ which must factor over some field extension of $\mathbb{Q}(s, t)$. In fact, these six quadratic polynomials all factor over the field $\mathbb{Q}(\sqrt{s}, \sqrt{t})$. For example, two of the $J_{i}$ contain the polynomial $(s-1) k^{2}-2 k l-l^{2}$, which is the product

$$
((\sqrt{s}+1) k+l)((\sqrt{s}-1) k-l) .
$$

For each ideal $J_{i}$, the factorization of the quadratic polynomial induces a factorization of $J_{i}$ into two ideals $J_{i 1}$ and $J_{i 2}$. Inspecting a Gröbner basis for each ideal shows that each defines a plane conic in $\mathbb{P}^{9}$. Thus, over the field $\mathbb{Q}(\sqrt{s}, \sqrt{t}), J$ defines 12 plane conics.

Theorem 12 is a consequence of the following two observations:

(1) The factorization of $J$ gives 12 distinct components for all values of the parameters $s, t$ satisfying (2.6).

(2) The map $\varphi$ does not vanish identically on any of the components $\mathcal{V}\left(J_{i j}\right)$ for values of the parameters $s, t$ satisfying (2.6).

By (1), no component of $J$ is empty for any $s, t$ satisfying (2.6) and thus, for every asymmetric $(2,2)$-curve $C$, there is a quadric $Q$ with $\varphi(Q)=C$. Also by (1), $J$ has exactly 12 components with each a plane conic, for any $s, t$ satisfying (2.6), and by (2), $\mathcal{V}(J)=\overline{\varphi^{-1}(C)}$.

\section{Symmetric Smooth (2, 2)-Curves}

We investigate smooth curves $C$ whose double points in the ramified fibers over $\ell_{1}$ have only two distinct projections to $\ell_{2}$. Assume that the ramification is at the points $[w, x]=[1,1],[1,-1],[1, s]$, and at $[1,-s]$, for some $s \in \mathbb{C} \backslash\{0, \pm 1\}$ with the double points in the fibers at $[y, z]=[1,0]$ for the first two and at $[0,1]$ for the second two. Since the points $[1,1],[1,-1],[1, s]$, and $[1,-s]$ have cross ratio

$$
\left[\frac{1+s}{1-s}, \frac{1-s}{1+s}\right]=\left[1, \frac{(1-s)^{2}}{(1+s)^{2}}\right],
$$

we see that all cross ratios in $\mathbb{P}^{1} \backslash\{[1,0],[0,1],[1,1]\}$ are obtained for some $s \in$ $\mathbb{C} \backslash\{0, \pm 1\}$. Thus our choice of ramification results in no loss of generality.

As in Section 2, these conditions give equations on the coefficients $c_{i j}$ of the general $(2,2)$-curve $(2.3)$ :

$$
\begin{aligned}
& c_{00}+c_{10}+c_{20}=0, \quad c_{01}+c_{11}+c_{21}=0, \\
& c_{01}-c_{11}+c_{21}=0, \quad c_{02}+c_{12} s+c_{22} s^{2}=0, \\
& c_{02}-c_{12} s+c_{22} s^{2}=0, \quad c_{01}-c_{11} s+c_{21} s^{2}=0 \text {. } \\
& \begin{array}{r}
c_{00}-c_{10}+c_{20}=0 \\
c_{01}+c_{11} s+c_{21} s^{2}=0,
\end{array}
\end{aligned}
$$


These equations have the following consequences:

$$
0=c_{21}=c_{01}=c_{12}=c_{11}=c_{10}=c_{02}+c_{22} s^{2}=c_{00}+c_{20} .
$$

Hence after normalizing by setting $c_{20}=1$, the $(2,2)$-form (2.3) becomes

$$
\left(x^{2}-w^{2}\right) y^{2}+c_{22}\left(x^{2}-s^{2} w^{2}\right) z^{2} .
$$

While the choice of ramification points $[1,1],[1,-1],[1, s],[1,-s]$ fixes the parametrization of $\ell_{1}$, the double points in the fibers of $[1,0]$ and $[0,1]$ do not fix the parametrization of $\ell_{2}$. Thus we are still free to scale the $z$-coordinate. We normalize this equation setting $c_{22}= \pm 1$. We do not simply set $c_{22}=1$ because that misses an important real form of the polynomial. This normalization gives

$$
\left(x^{2}-w^{2}\right) y^{2} \pm\left(x^{2}-s^{2} w^{2}\right) z^{2}=\left(y^{2} \pm z^{2}\right) x^{2}-\left(y^{2} \pm s^{2} z^{2}\right) w^{2} .
$$

This shows the equation to be symmetric under the involution $[w, x] \leftrightarrow[\sqrt{\mp 1} z, y]$. This symmetry is the source of our terminology for the two classes of $(2,2)$-curves. Also, if $s \notin\{ \pm 1,0\}$, then this is the equation of a smooth $(2,2)$-curve. With the choice of sign $(-)$, which we call the curve $C(s)$.

Note that (4.1) is real if $s$ either is real or is purely imaginary $(s \in \mathbb{R} \sqrt{-1})$. We complete the proof of Theorem 1 with the following result for symmetric $(2,2)$-curves.

Theorem 14. For each $s \in \mathbb{C} \backslash\{ \pm 1,0\}$, the closure of the fiber $\varphi^{-1}(C(s))$ consists of 12 distinct plane conics. When $s \in \mathbb{R}$ or $s \in \mathbb{R} \sqrt{-1}$ and we use the real form of (4.1) with the plus sign $(+)$, then exactly 4 of these 12 components will be real. If we use the real form of (4.1) with the minus sign (-), then if $s \in \mathbb{R}$, all 12 components will be real, but if $s \in \mathbb{R} \sqrt{-1}$, then exactly 4 of these 12 components will be real.

Proof. Our proof follows the proof of Theorem 12 almost exactly, but with significant simplifications and a case analysis. Unlike the proof described in Section 3, we do not give annotated SINGULAR code in an appendix, but rather supply such annotated SINGULAR code on a web page. ${ }^{1}$

The outline is as before, except that we work over the ring of parameters $\mathbb{Q}(s)$, and find no extraneous components when we factor the ideal into components. We formulate this as a system of equations, remove the same three excess components, and then factor the resulting ideal. We do this calculation four times, once for each choice of sign $( \pm)$ in (4.1), and for $s \in \mathbb{R}$ and $s \in \mathbb{R} \sqrt{-1}$. Examining the output proves the result.

We consider in some detail four cases of the geometry studied in Section 2, which correspond to the four real cases of Theorem 14. As in Section 2, let $\ell_{1}$ be the $x$-axis and let $\ell_{2}$ be the $y z$-line at infinity. Viewed in $\mathbb{R}^{3}$, lines transversal to $\ell_{1}$ and $\ell_{2}$ are the set of lines perpendicular to the $x$-axis. For a transversal line $\ell$, the coordinates $[y, z]$ of the point $\ell \cap \ell_{2}$ can be interpreted as the slope of $\ell$ in the two-dimensional plane orthogonal to the $x$-axis.

\footnotetext{
${ }^{1}$ http: //www. math. umass.edu/ sottile/pages/212s/
} 
Consider real quadrics given by an equation of the form

$$
x^{2}+\left(y-y_{0}\right)^{2} \pm z^{2}=1 .
$$

The quadrics with the plus (+) sign are spheres with center $\left(0, y_{0}, 0\right)^{\mathrm{T}}$ and radius 1 , and those with the minus (-) sign are hyperboloids of one sheet. When $\left|y_{0}\right|>1$ the quadric does not meet the $x$-axis. We look at four families of such quadrics: spheres and hyperboloids that meet and do not meet the $x$-axis. We remark that quadrics which are tangent to the $x$-axis give singular $(2,2)$-curves, as shown in Theorem 11.

First, consider the resulting $(2,2)$-curve

$$
\left(x^{2}-w^{2}\right) y^{2} \pm\left(x^{2}-\left(1-y_{0}^{2}\right) w^{2}\right) z^{2} .
$$

Thus we see that these correspond to the case $s=\sqrt{1-y_{0}^{2}}$ in the parametrization of symmetric (2,2)-curves given above (4.1), while in (4.2) and (4.1) the signs ( \pm ) correspond.

Figures 1 and 2 display pictures of these four quadrics, together with the $x$-axis, some tangents perpendicular to the $x$-axis, and the curve on the quadric where the lines are tangent.

Remark 15. For each of the spheres, there is another sphere of radius $r$ which leads to the same envelope, namely, the one with center $\left(0,-y_{0}, 0\right)^{\mathrm{T}}$.

The ramification of the $(2,2)$-curve of tangents perpendicular to the $x$-axis is evident from Figs. 1 and 2. When $x= \pm 1$, there is a single tangent line; this line has slope $[y, z]=[1,0]$, i.e., it is a horizontal line. When $x= \pm \sqrt{1-y_{0}^{2}}$, there is a single tangent line, which is vertical (i.e., which has slope $[y, z]=[0,1])$. Figures 1 and 2 depict these lines in case they are real. In Fig. 1 we have $\left|y_{0}\right|>1$, and hence the vertical tangent lines are complex. All other values of $x$ give two lines perpendicular to the $x$-axis and tangent to the quadric, but some have imaginary slope.

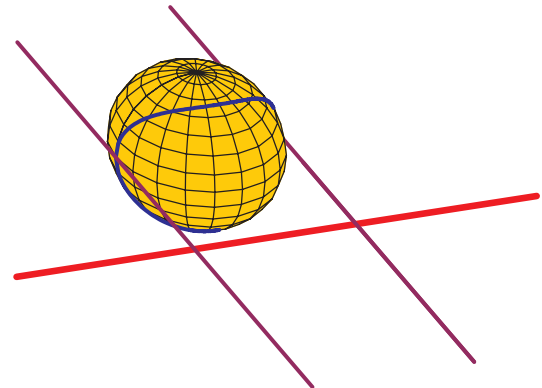

(a)

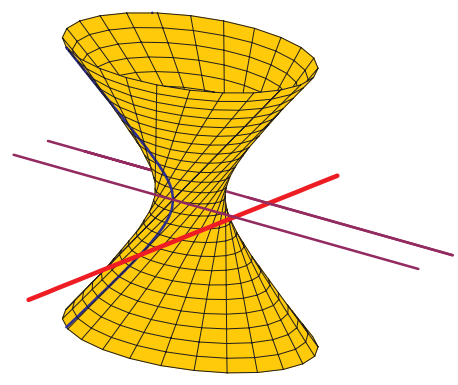

(b)

Fig. 1. Real quadrics not meeting the $x$-axis. 


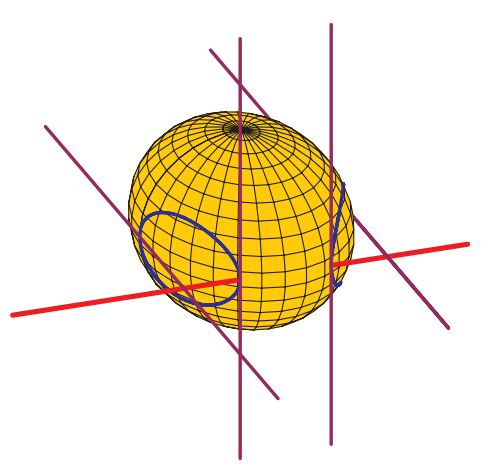

(a)

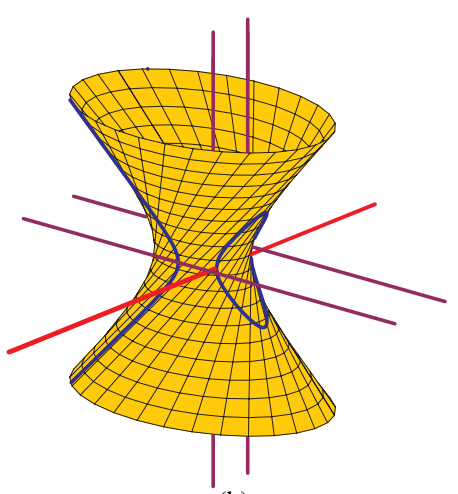

(b)

Fig. 2. Real quadrics meeting the $x$-axis.

The difference in the number of real components of the fiber $\varphi^{-1}(C(s))$ noted in Theorem 14 can be seen in these examples. The spheres and hyperboloid displayed together are isomorphic under the change of coordinates $z \mapsto \sqrt{-1} \cdot z$, which interchanges the transversal tangents of purely imaginary slope for one quadric with the real transversal tangents of the other and corresponds to the different signs \pm in (4.2) and (4.1).

For the sphere of Fig. 1, only 4 of the 12 families are real. One consists of ellipsoids, including the original sphere, one of hyperboloids of two sheets, and two of hyperboloids of one sheet. Since a hyperboloid of two sheets can be seen as an ellipsoid meeting the plane at infinity in a conic, we see there are two families of ellipsoids and two of hyperboloids. In Fig. 3 we display one quadric from each family (except the family of the sphere), together with the original sphere, the $x$-axis, and the curve on the quadric where the lines perpendicular to the $x$-axis are tangent to the quadric.
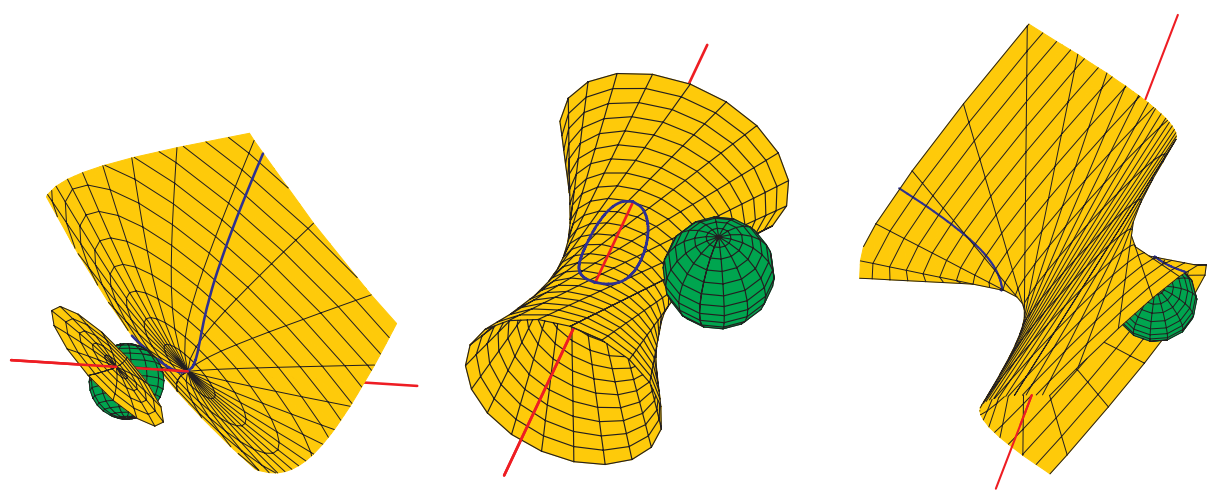

Fig. 3. The other three families. 
Similarly, the hyperboloid of Fig. 1 has only 4 of its 12 families real with two families of ellipsoids and two of hyperboloids. The sphere of Fig. 2 has only 4 of its 12 families real, and all four contain ellipsoids. In contrast, the hyperboloid of Fig. 2 has all 12 of its families real, and they contain only hyperboloids of one sheet.

Many more pictures (in color) are found on the web page accompanying this article.

\section{Transversals to Two Lines and Tangents to Two Spheres}

We solve the original question of configurations of two lines and two spheres for which there are infinitely many real transversals to the two lines that are also tangent to both spheres. While general quadrics are naturally studied in projective space $\mathbb{P}^{3}$, spheres naturally live in (the slightly more restricted) affine space $\mathbb{R}^{3}$. As noted in Section 2, we treat only skew lines. There are two cases to consider: either the two lines are in $\mathbb{R}^{3}$ or one lies in the plane at infinity. We work throughout over the real numbers.

\subsection{Lines in Affine Space $\mathbb{R}^{3}$}

The complete geometric characterization of configurations where the lines lie in $\mathbb{R}^{3}$ is stated in the following theorem and illustrated in Fig. 4.

Theorem 16. Let $S_{1}$ and $S_{2}$ be two distinct spheres and let $\ell_{1}$ and $\ell_{2}$ be two skew lines in $\mathbb{R}^{3}$. There are infinitely many lines that meet $\ell_{1}$ and $\ell_{2}$ and are tangent to $S_{1}$ and $S_{2}$ in exactly the following cases:

(1) The spheres $S_{1}$ and $S_{2}$ are tangent to each other at a point $p$ which lies on one line, and the second line lies in the common tangent plane to the spheres at the point $p$. The pencil of lines through $p$ that also meet the second line is exactly the set of common transversals to $\ell_{1}$ and $\ell_{2}$ that are also tangent to $S_{1}$ and $S_{2}$.

(2) The lines $\ell_{1}$ and $\ell_{2}$ are each tangent to both $S_{1}$ and $S_{2}$, and they are images of each other under a rotation about the line connecting the centers of $S_{1}$ and $S_{2}$.

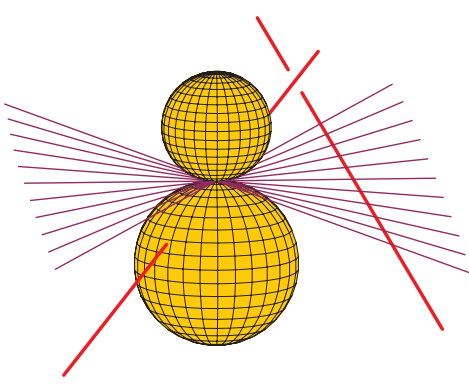

(1)

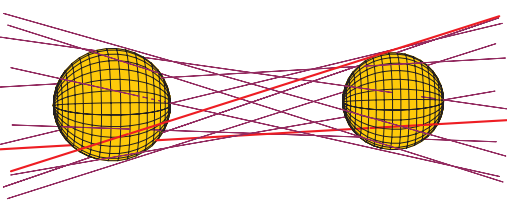

(2)

Fig. 4. Examples from Theorem 16. 
If we rotate $\ell_{1}$ about the line connecting the centers of the spheres, it sweeps out a hyperboloid of one sheet. One of its rulings contains $\ell_{1}$ and $\ell_{2}$, and the lines in the other ruling are tangent to $S_{1}$ and $S_{2}$ and meet $\ell_{1}$ and $\ell_{2}$, except for those that are parallel to one of them.

Let $\ell_{1}$ and $\ell_{2}$ be two skew lines. The class of spheres is not invariant under the set of projective linear transformations, but rather under the group generated by rotations, translations, and scaling the coordinates. Thus we can assume that

$\ell_{1}=\left\{\left(\begin{array}{l}0 \\ 0 \\ 1\end{array}\right)+x\left(\begin{array}{l}1 \\ \delta \\ 0\end{array}\right): x \in \mathbb{R}\right\}, \quad \ell_{2}=\left\{\left(\begin{array}{c}0 \\ 0 \\ -1\end{array}\right)+z\left(\begin{array}{c}-1 \\ -\delta \\ 0\end{array}\right): z \in \mathbb{R}\right\}$

for some $\delta \in \mathbb{R} \backslash\{0\}$. As before, there is a one-to-one correspondence between lines meeting $\ell_{1}$ and $\ell_{2}$ and pairs $(x, z) \in \mathbb{R}^{2}$. The transversal corresponding to a pair $(x, z)$ passes through the points $(x, \delta x, 1)^{\mathrm{T}}$ and $(z,-\delta z,-1)^{\mathrm{T}}$, and has Plücker coordinates

$$
(x-z, \delta(x+z), 2,2 \delta x z, x+z, \delta(x-z))^{\mathrm{T}} .
$$

Let $S_{1}$ have center $(a, b, c)^{\mathrm{T}}$ and radius $r$. By Proposition 2 and (1.4), the transversals tangent to $S_{1}$ are parametrized by a curve $C_{1}$ of degree 4 with equation

$$
\begin{aligned}
0= & 4 \delta^{2} x^{2} z^{2}+4 \delta(b-a \delta) x^{2} z+\left((b-a \delta)^{2}+\left(1+\delta^{2}\right)\left((1+c)^{2}-r^{2}\right)\right) x^{2} \\
& -4 \delta(b+a \delta) x z^{2}+2\left(\left(r^{2}-c^{2}\right)\left(1-\delta^{2}\right)+\left(1-b^{2}\right)+\delta^{2}\left(a^{2}-1\right)\right) x z \\
& -4(1+c)(a+b \delta) x+\left((b+a \delta)^{2}+\left(1+\delta^{2}\right)\left((1-c)^{2}-r^{2}\right)\right) z^{2} \\
& +4(c-1)(a-b \delta) z+4\left(a^{2}+b^{2}-r^{2}\right) .
\end{aligned}
$$

This is a dehomogenized version of the bihomogeneous equation $(2.3)$ of bidegree $(2,2)$. Note also that the curve $C_{1}$ is defined over our ground field $\mathbb{R}$. The transversals to $\ell_{1}$ and $\ell_{2}$ tangent to $S_{2}$ are parametrized by a similar curve $C_{2}$. There are infinitely many lines which meet $\ell_{1}$ and $\ell_{2}$ and are tangent to $S_{1}$ and $S_{2}$ if and only if the curves $C_{1}$ and $C_{2}$ have a common component. That is, if and only if the associated polynomials share a common factor. We first rule out the case when the curves are irreducible.

Lemma 17. The curve $C_{1}$ in (5.1) determines the sphere $S_{1}$ uniquely.

Proof. Given the curve (5.1), we can rescale the equation such that the coefficient of $x^{2} z^{2}$ is $4 \delta^{2}$. From the coefficients of $x^{2} z$ and $x z^{2}$ we can determine $a$ and $b$, and then from the coefficients of $x^{2}$ and $z^{2}$ we can determine $c$ and $r$.

Remark 18. By Remark 15, Lemma 17 does not hold if the lines are allowed to live in projective space $\mathbb{P}^{3}$. We come back to this in Section 5.2.

By Lemma 17, there can be infinitely many common transversals to $\ell_{1}$ and $\ell_{2}$ that are tangent to two spheres only if the curves $C_{1}$ and $C_{2}$ are reducible. In particular, this rules out cases (1) and (2) of Lemma 3. Our classification of factors of $(2,2)$-forms in 
Lemma 3 gives the following possibilities for the common irreducible factors (over $\mathbb{R}$ ) of $C_{1}$ and $C_{2}$, up to interchanging $x$ and $z$. Either the factor is a cubic (the dehomogenization of a $(2,1)$-form), or it is linear in $x$ and $z$ (the dehomogenization of a $(1,1)$-form), or it is linear in $x$ alone (the dehomogenization of a $(1,0)$-form). There is the possibility that the common factor will be an irreducible (over $\mathbb{R}$ ) quadratic polynomial in $x$ (coming from a $(2,0)$-form), but then this component will have no real points, and thus contributes no common real tangents.

We rule out the possibility of a common cubic factor, showing that if $C_{1}$ factors as $x-x_{0}$ and a cubic, then the cubic still determines $S_{1}$. The vector $\left(-\delta,-1, \delta x_{0}\right)^{\mathrm{T}}$ is perpendicular to the plane through $\left(x_{0}, \delta x_{0}, 1\right)^{\mathrm{T}}$ and $\ell_{2}$, so the center of $S_{1}$ will be $\left(x_{0}, \delta x_{0}, 1\right)^{\mathrm{T}}+\lambda\left(-\delta,-1, \delta x_{0}\right)^{\mathrm{T}}$ for some non-zero $\lambda \in \mathbb{R}$. Thus $r^{2}=\lambda^{2}\left(1+\delta^{2}+\delta^{2} x_{0}^{2}\right)$. Substituting this into (5.1) and dividing by $\left(x-x_{0}\right)$ we obtain the equation of the cubic:

$$
\begin{aligned}
0= & \delta^{2} x z^{2}+\delta\left(\delta^{2}-1\right) \lambda x z+\left(1+\delta^{2}\left(1-\lambda^{2}\right)+\delta \lambda\left(1+\delta^{2}\right) x_{0}\right) x \\
& +\delta\left(\lambda\left(1+\delta^{2}\right)-\delta x_{0}\right) z^{2}+\delta\left(\delta^{2}-1\right) \lambda x_{0} z+4 \delta \lambda+\left(\delta^{2} \lambda^{2}-\delta^{2}-1\right) x_{0} .
\end{aligned}
$$

Given only this curve, we can rescale its equation so that the coefficient of $x z^{2}$ is $\delta^{2}$, then if $\delta \neq \pm 1$, we can uniquely determine $\lambda, x_{0}$, and therefore $S_{1}$, too, from the coefficients of $x z$ and $x$.

The uniqueness is still true if $\delta= \pm 1$. Assume that $\delta=1$. Then (5.2) reduces to

$$
x z^{2}+\left(2 \lambda-x_{0}\right) z^{2}+\left(2-\lambda^{2}+2 \lambda x_{0}\right) x+4 \lambda+\left(\lambda^{2}-2\right) x_{0}=0 .
$$

Set $\alpha:=2 \lambda-x_{0}, \beta:=2-\lambda^{2}+2 \lambda x_{0}$, and $\gamma:=4 \lambda+\left(\lambda^{2}-2\right) x_{0}$. We can solve for $\lambda$ and $x_{0}$ in terms of $\alpha$ and $\beta$,

$$
\lambda=\frac{\alpha \pm \sqrt{\alpha^{2}+3 \beta-6}}{3}, \quad x_{0}=\frac{-\alpha \pm 2 \sqrt{\alpha^{2}+3 \beta-6}}{3} .
$$

(We take the same sign of the square root in both cases.) If we substitute these values into the formula for $\gamma$, we see that the two possible values of $\gamma$ coincide if and only if $\alpha^{2}+3 \beta-6=0$, in which case there is only one solution for $\lambda$ and $x_{0}$, so $\alpha, \beta$, and $\gamma$ always determine $\lambda$ and $x_{0}$ and hence $S_{1}$ uniquely. The case $\delta=-1$ is similar.

We now are left only with the cases when $C_{1}$ and $C_{2}$ contain a common factor of the form $x-x_{0}$ or $x z+s x+t z+u$. Suppose the common factor is $x-x_{0}$. Then any line through $p:=\left(x_{0}, \delta x_{0}, 1\right)^{\mathrm{T}}$ and a point of $\ell_{2}$ is tangent to $S_{1}$. This is only possible if the sphere $S_{1}$ is tangent to the plane through $p$ and $\ell_{2}$ at the point $p$. We conclude that if $C_{1}$ and $C_{2}$ have the common factor $x-x_{0}$, then the spheres $S_{1}$ and $S_{2}$ are tangent to each other at the point $p=\left(x_{0}, \delta x_{0}, 1\right)^{\mathrm{T}}$ lying on $\ell_{1}$ and $\ell_{2}$ lies in the common tangent plane to the spheres at the point $p$. This is case (1) of Theorem 16.

Suppose now that $C_{1}$ and $C_{2}$ have a common irreducible factor $x z+s x+t z+u$. We can solve the equation $x z+s x+t z+u=0$ uniquely for $z$ in terms of $x$ for general values of $x$, or for $x$ in terms of $z$ for general values of $z$, this gives rise to an isomorphism $\phi$ between the projectivizations of $\ell_{1}$ and $\ell_{2}$. The lines connecting $q$ and $\phi(q)$ as $q$ runs through the points of $\ell_{1}$ sweep out a hyperboloid of one sheet. The lines $\ell_{1}$ and $\ell_{2}$ are contained in one ruling, and the lines meeting both of them and tangent to $S_{1}$ are the lines in the other ruling. 
Lemma 19. Let $H \subset \mathbb{R}^{3}$ be a hyperboloid of one sheet. If all lines in one of its rulings are tangent to a sphere $S$, then $H$ is a hyperboloid of revolution, the center of the sphere $S$ is on the axis of rotation and $S$ is tangent to $H$.

Proof. We can choose Cartesian coordinates such that $H$ has equation $x^{2} / \alpha^{2}+y^{2} /$ $\beta^{2}-z^{2} / \gamma^{2}=1$ for some positive real numbers $\alpha, \beta, \gamma$. Let the sphere have center $(A, B, C)^{\mathrm{T}}$ and radius $R$. The set of points of the form $(\alpha, \lambda \beta, \lambda \gamma)^{\mathrm{T}},(-\alpha,-\lambda \beta, \lambda \gamma)^{\mathrm{T}}$, $(-\lambda \alpha, \beta, \lambda \gamma)^{\mathrm{T}}$, and $(\lambda \alpha,-\beta, \lambda \gamma)^{\mathrm{T}}$ as $\lambda$ runs through $\mathbb{R}$ form four lines in one of the rulings. Since the two rulings are symmetric, we only need to deal with one of them.

The sphere $S$ is tangent to a line if and only if the distance of the line from the center of $S$ is $R$. The condition that $(A, B, C)^{\mathrm{T}}$ must be at the same distance from the first two lines gives the equation

$$
\alpha\left(\beta^{2}+\gamma^{2}\right) A+\beta \gamma B C=0,
$$

the equality of distances from the other two lines gives

$$
\beta\left(\alpha^{2}+\gamma^{2}\right) B-\alpha \gamma A C=0 .
$$

Since $\alpha, \beta, \gamma>0$, the common solutions of these equations have $A=B=0$. Using this information, the equality of the distances from the first and third lines gives $\alpha=\beta$, or $C= \pm \sqrt{\left(\alpha^{2}+\gamma^{2}\right)\left(\beta^{2}+\gamma^{2}\right) / \gamma}$. To eliminate this second possibility, consider two more lines in the same ruling, the points of the form

$$
\left(\alpha \frac{1-\lambda}{\sqrt{2}}, \beta \frac{1+\lambda}{\sqrt{2}}, \lambda \gamma\right)^{\mathrm{T}} \text { and }\left(\alpha \frac{1+\lambda}{\sqrt{2}}, \beta \frac{-1+\lambda}{\sqrt{2}}, \lambda \gamma\right)^{\mathrm{T}}
$$

as $\lambda$ runs through $\mathbb{R}$. The equality of distances from these two lines together with $A=$ $B=0$ gives $\alpha=\beta$ or $C=0$.

Therefore the only case when $(A, B, C)^{\mathrm{T}}$ can be at the same distance from all lines in one ruling of $H$ is when $\alpha=\beta$, i.e., $H$ is a hyperboloid of revolution about the $z$-axis, and $(A, B, C)^{\mathrm{T}}$ lies on the $z$-axis. In this case it is obvious that $(A, B, C)^{\mathrm{T}}$ is at the same distance from all the lines contained in $H$, and these lines are tangent to $S$ if and only if $S$ is tangent to $H$.

By this lemma, the hyperboloid swept out by the lines meeting $\ell_{1}$ and $\ell_{2}$ and tangent to $S_{1}$ is a hyperboloid of revolution with the center of $S_{1}$ on the axis of rotation. Furthermore, $\ell_{1}$ and $\ell_{2}$ are lines in one of the rulings of the hyperboloid, therefore they are images of each other under suitable rotation about the axis, the images of $\ell_{1}$ sweep out the whole hyperboloid, and $\ell_{1}, \ell_{2}$ are both tangent to $S_{1}$. Applying the lemma to $S_{2}$ shows that the center of $S_{2}$ is also on the axis of rotation and $\ell_{1}, \ell_{2}$ are both tangent to $S_{2}$. We cannot have $S_{1}$ and $S_{2}$ concentric, therefore the axis of rotation is the line through their centers. This is exactly case (2) of Theorem 16, and we have completed its proof.

\subsection{Lines in Projective Space}

We give the complete geometric characterization of configurations in real projective space where the line $\ell_{2}$ lies in the plane at infinity. 
Theorem 20. Let $S_{1}$ and $S_{2}$ be two distinct spheres and let $\ell_{1}$ lie in $\mathbb{R}^{3}$ with $\ell_{2}$ a line at infinity skew to $\ell_{1}$. There are infinitely many lines that meet $\ell_{1}$ and $\ell_{2}$ and are tangent to $S_{1}$ and $S_{2}$ in exactly the following cases:

(1) The spheres $S_{1}$ and $S_{2}$ are tangent to each other at a point $p$ which lies on $\ell_{1}$, and $\ell_{2}$ is the line at infinity in the common tangent plane to the spheres at the point $p$. The pencil of lines through $p$ that lie in this tangent plane are exactly the common transversals to $\ell_{1}$ and $\ell_{2}$ that are also tangent to $S_{1}$ and $S_{2}$.

(2) Any line meeting $\ell_{1}$ and $\ell_{2}$ is perpendicular to $\ell_{1}$ and $S_{1}$ and $S_{2}$ are related to each other by multiplication by -1 in the directions perpendicular to $\ell_{1}$. Thus when $\ell_{1}$ is not tangent to the spheres, we are in exactly the situation of Remark 15 of Section 4 as shown in Figs. 1(a) and 2(a).

Proof. Let $\Pi$ be any plane passing through a point of $\ell_{1}$ and containing $\ell_{2}$. Then common transversals to $\ell_{1}$ and $\ell_{2}$ are lines meeting $\ell_{1}$ that are parallel to $\Pi$. Choose a Cartesian coordinate system in $\mathbb{R}^{3}$ such that $\ell_{1}$ is the $x$-axis. Suppose that $S_{1}$ has center $(a, b, c)^{\mathrm{T}}$ and radius $r$. Let $u=\left(u_{1}, u_{2}, 0\right)^{\mathrm{T}}$ and $v=\left(v_{1}, 0, v_{3}\right)^{\mathrm{T}}$ be vectors with $u_{2} \neq 0$ and $v_{3} \neq 0$ parallel to $\Pi$. Such vectors exist as $\ell_{1}$ and $\ell_{2}$ are skew. A common transversal to $\ell_{1}$ and $\ell_{2}$ is determined by the intersection point $(x, 0,0)^{\mathrm{T}}$ with $\ell_{1}$ and a direction vector corresponding to the intersection point with $\ell_{2}$, which can be written as $u+z v$ for some $z \in \mathbb{R}$, unless it is parallel to $v$. Since $S_{1}$ has at most two tangent lines which meet $\ell_{1}$ that are parallel to $v$, so by omitting these we are not losing an infinite family of common transversals/tangents.

The transversals that are tangent to $S_{1}$ are parametrized by a curve $C_{1}$ in the $x z$-plane with equation

$$
\begin{aligned}
0= & v_{3}^{2} x^{2} z^{2}+u_{2}^{2} x^{2}+2 v_{3}\left(c v_{1}-a v_{3}\right) x z^{2}+2\left(b u_{2} v_{1}+c u_{1} v_{3}\right) x z \\
& +2 u_{2}\left(b u_{1}-a u_{2}\right) x+\left(\left(b^{2}+c^{2}-r^{2}\right) v_{1}^{2}-2 a c v_{1} v_{3}+\left(a^{2}+b^{2}-r^{2}\right) v_{3}^{2}\right) z^{2} \\
& +2\left(\left(b^{2}+c^{2}-r^{2}\right) u_{1} v_{1}-a c u_{1} v_{3}-b u_{2}\left(a v_{1}+c v_{3}\right)\right) z \\
& +\left(\left(b^{2}+c^{2}-r^{2}\right) u_{1}^{2}-2 a b u_{1} u_{2}+\left(a^{2}+c^{2}-r^{2}\right) u_{2}^{2}\right)
\end{aligned}
$$

The transversals tangent to $S_{2}$ are parametrized by a similar curve $C_{2}$. There are infinitely many lines that meet $\ell_{1}$ and $\ell_{2}$ and are tangent to $S_{1}$ and $S_{2}$ if and only if $C_{1}$ and $C_{2}$ have a common non-empty real component.

It is easy to see from the coefficients of $x z^{2}, x z$, and $x$ and the constant term that if $u_{1} \neq 0$ or $v_{1} \neq 0$, then $C_{1}$ determines $a, b, c$, and $r^{2}$ and therefore $S_{1}$ uniquely, so if $C_{1}$ is irreducible and $u_{1} \neq 0$ or $v_{1} \neq 0$, then there cannot be infinitely many common transversals that are tangent to $S_{1}$ and $S_{2}$.

Assume now that $u_{1}=v_{1}=0$, this is equivalent to the plane $\Pi$ being perpendicular to $\ell_{1}$. From the coefficient of $x$ we can determine $a$, and then from the coefficients of $z^{2}, z$, and the constant term we can calculate the quantities $\alpha=c^{2}-r^{2}, \beta=b c$, and $\gamma=b^{2}-r^{2}$. The equation $\left(\alpha+r^{2}\right)\left(\gamma+r^{2}\right)-\beta^{2}=0$ is a quadratic equation for $r^{2}$ with solutions

$$
r^{2}=\frac{1}{2}\left(-\alpha-\gamma \pm \sqrt{(\alpha-\gamma)^{2}+4 \beta^{2}}\right) .
$$

Only the larger root is feasible, even when both are positive, since both $\alpha+r^{2}=c^{2}$ and $\gamma+r^{2}=b^{2}$ must be non-negative. Hence $r^{2}$, and thus $b^{2}$ and $c^{2}$, are uniquely 
determined. The values of $b^{2}, b c$, and $c^{2}$ determine two possible pairs $(b, c)$ which are negatives of each other. This is exactly case (2) of the theorem. In fact, this case is illustrated by Figs. 1(a) and 2(b).

We now consider the cases when $C_{1}$ is reducible. As in the proof of Theorem 16, we need only consider cubics and factors of the form $x z+s x+t z+u, x-x_{0}$, and $z-z_{0}$.

Assume that $C_{1}$ has a component with equation $x z+s x+t z+u$. As described in the proof of Theorem 16, this establishes an isomorphism between the projectivizations of $\ell_{1}$ and $\ell_{2}$. The lines connecting the corresponding points of the projectivizations of $\ell_{1}$ and $\ell_{2}$ sweep out a hyperbolic paraboloid. However, the lines in one ruling of the hyperbolic paraboloid cannot all be tangent to a sphere, therefore this case cannot occur.

Likewise, the factor $z-z_{0}$ cannot appear, since it would mean that all the lines through a point of $\ell_{1}$ parallel to a certain direction are tangent to $S_{1}$, which is clearly impossible.

Consider the case where the equation of $C_{1}$ has a factor of $x-x_{0}$. As we saw in the proof of Theorem 16, $\ell_{1}$ meets the sphere $S_{1}$ at the point $p:=\left(x_{0}, 0,0\right)^{\mathrm{T}}$, and $\ell_{2}$ lies in the tangent plane to $S_{1}$ at $p$, and so this tangent plane is parallel to $\Pi$.

If $x-x_{0}$ is a factor of $C_{2}$, too, then $C_{2}$ passes through $p$ and its tangent plane there is also parallel to $\Pi$, so we have case (1) of the theorem.

To finish the proof we investigate what happens if the common component of $C_{1}$ and $C_{2}$ is the cubic obtained from $C_{1}$ after removing the line $x-x_{0}=0$.

The center of $S_{1}$ has coordinates $\left(x_{0}+\mu u_{2} v_{3},-\mu u_{1} v_{3},-\mu u_{2} v_{1}\right)^{\mathrm{T}}$ for some $\mu \in \mathbb{R}$, since $S_{1}$ passes through $\left(x_{0}, 0,0\right)^{\mathrm{T}}$ and its tangent plane there is parallel to $\Pi$, and we have $r^{2}=\mu^{2}\left(u_{1}^{2} v_{3}^{2}+u_{2}^{2} v_{1}^{2}+u_{2}^{2} v_{3}^{2}\right)$. Substituting this into (5.3) we obtain the equation of the remaining cubic,

$$
\begin{aligned}
v_{3}^{2} x z^{2}+ & u_{2}^{2} x-v_{3}\left(x_{0} v_{3}+2 \mu u_{2}\left(v_{1}^{2}+v_{3}^{2}\right)\right) z^{2} \\
& -4 \mu u_{1} u_{2} v_{1} v_{3} z-u_{2}\left(x_{0} u_{2}+2 \mu v_{3}\left(u_{1}^{2}+u_{2}^{2}\right)\right)=0 .
\end{aligned}
$$

If $u_{1} \neq 0$ or $v_{1} \neq 0$, then from the coefficients of this curve we can determine $x_{0}$ and $\mu$, hence $S_{1}$ uniquely, so $C_{1}$ and $C_{2}$ cannot have a common cubic component. If $u_{1}=v_{1}=0$, then the above equation factorizes as

$$
\left(x-\left(2 \mu u_{2} v_{3}+x_{0}\right)\right)\left(v_{3}^{2} z^{2}+u_{2}^{2}\right)=0,
$$

so if $C_{2}$ contains the curve defined by this equation, then the line $x-\left(2 \mu u_{2} v_{3}+x_{0}\right)=0$ is a common component of both $C_{1}$ and $C_{2}$, which is a case we have already dealt with.

\section{Acknowledgments}

We thank Dan Grayson and Mike Stillman, who, as editors for the book Computations in Algebraic Geometry with Macaulay 2 introduced us to the typography used in the Appendix. 


\section{Appendix. Calculations from Section 3}

We describe the computation of Section 3 in much more detail, giving a commentary on the SINGULAR file that accomplishes the computation and displaying its output. The input and output are displayed in typewriter font on separate lines and the output begins with the SINGULAR comment characters $(/ /)$.

The library primdec. lib contains the function sat for saturating ideals, and the option redSB forces SINGULAR to work with reduced Gröbner (standard) bases.

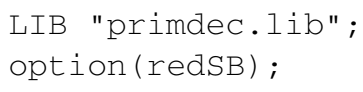

We initialize our ring.

ring $R=0,(s, t, a, b, c, d, e, f, g, h, k, 1),(d p(2), \quad d p(10))$;

The underlying coefficient field has characteristic 0 (so it is $\mathbb{Q}$ ) and variables $s, t, a, \ldots$, $k, l$, with a product term order chosen to simplify our analysis of the projection to $\mathbb{C}^{2}$, the space of parameters.

We consider the ideal generated by (3.5)

ideal $I=e l-g^{\wedge} 2$, ek-gf, ak-dc, ah-c^2;

and by the $2 \times 2$ minors of the coefficient matrix (3.6).

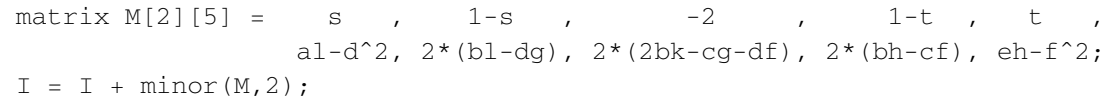

We check the dimension and degree (multiplicity) of the variety $\mathcal{V}(I)$, first computing a Gröbner basis for $I$.

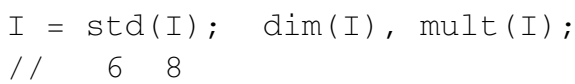

SINGULAR gives the dimension of $\mathcal{V}(I)$ in affine space $\mathbb{C}^{12}$. Since $I$ is homogeneous in the variables $a, b, \ldots, h, k, l$, we consider $\mathcal{V}(I)$ to be a subvariety of $\mathbb{P}^{9} \times \mathbb{C}^{2}$. Its dimension is one less than that of the corresponding affine variety. Thus $\mathcal{V}(I)$ has dimension 5 and degree 8.

In Section 3 we identified three spurious components of $\mathcal{V}(I)$ which we remove. The first and largest is the ideal of rank 1 quadrics, given by the $2 \times 2$ minors of the $4 \times 4$ symmetric matrix (3.3).

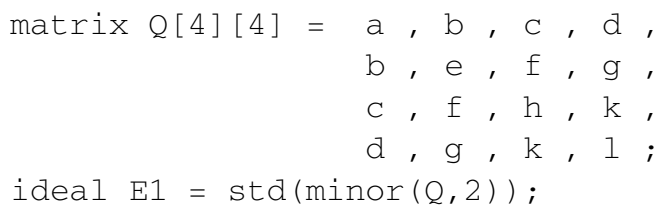

We remove this spurious component by computing the quotient ideal $\left(I: E_{1}\right)$.

$I=\operatorname{std}(q u o t i e n t(I, E 1)) ; \operatorname{dim}(I), \operatorname{mult}(I) ;$

// 520 
The other two spurious components describe rank 2 quadrics which are unions of two planes with intersection line $\ell_{1}$ or $\ell_{2}$.

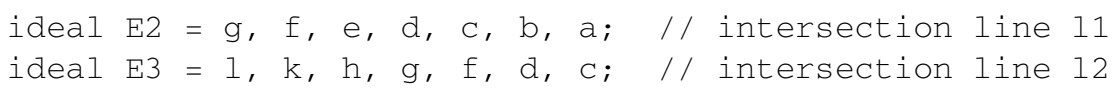

The corresponding components are not reduced; rather than take ideal quotients, we saturate the ideal $I$ with respect to these spurious ideals. The SINGULAR command sat for saturation returns a pair whose first component is a Gröbner basis of the saturation and the second is the saturation exponent. Here, both saturations have exponent 4 . We saturate $I$ with respect to $E_{2}$,

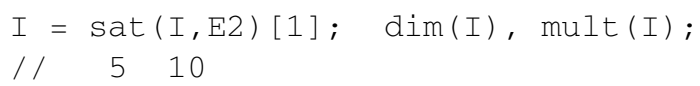

and then with respect to $E_{3}$.

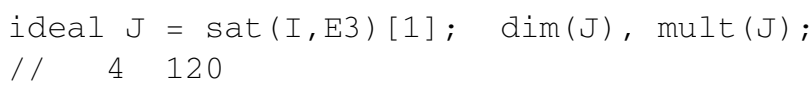

Thus we now have a variety $\mathcal{V}(J)$ of dimension 3 in $\mathbb{P}^{9} \times \mathbb{C}^{2}$. We check that it projects onto the space $\mathbb{C}^{2}$ of parameters by eliminating the variables $a, b, \ldots, h, k, l$ from $J$.

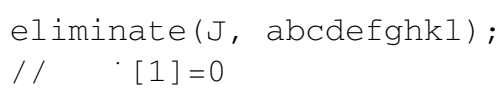

Since we obtain the zero ideal, the image of $\mathcal{V}(J)$ is Zariski dense in $\mathbb{C}^{2}[2$, Chapter 4 , Section 4]. However, the projection $\mathbb{P}^{9} \times \mathbb{C}^{2} \rightarrow \mathbb{C}^{2}$ is a closed map, so the image of $\mathcal{V}(J)$ is $\mathbb{C}^{2}$. Thus, for every smooth $(2,2)$-curve $C$ defined by $(2.5)$, there is a quadric whose transversal tangents are described by the curve $C$.

We now apply the factorization Gröbner basis algorithm facstd to $J$. The second argument of facstd is the list of non-zero constraints which are given by Theorem 7 .

ideal $\mathrm{L}=\mathrm{s}, \mathrm{t}, \mathrm{t}-1, \mathrm{~s}-1, \mathrm{~s}-\mathrm{t}$;

list $F=\operatorname{facstd}(\mathrm{J}, \mathrm{L})$;

SINGULAR computes seven factors

$\operatorname{size}(\mathrm{F})$;

// 7

Since $J$ and the seven factors $F_{1}, \ldots, F_{7}$ are radical ideals, this factorization can be verified by checking that that the following ideals $V_{1}$ and $V_{2}$ coincide. (This part of the calculation is archived on the web page accompanying this article.)

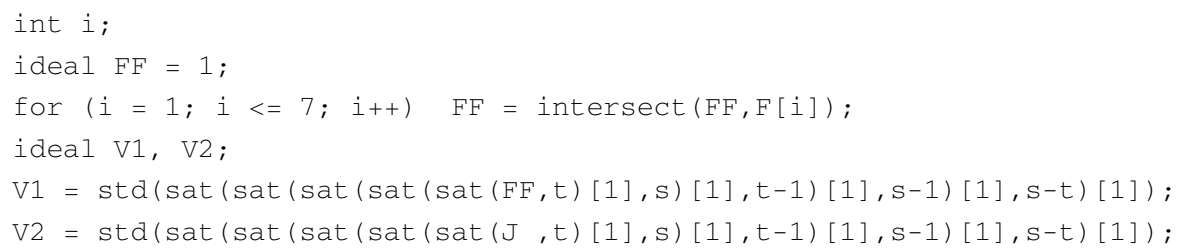


Note, in particular, that for any given explicit values of $s, t$ satisfying the non-zero conditions, the parametric factorization (in $s, t$ ) produced by facsta can be specialized to an explicit factorization.

We examine the ideals in the list $F$, working over the ring with parameters.

ring $S=(0, s, t),(a, b, c, d, e, f, g, h, k, l), l p ;$ short $=0$;

First, the ideal $J$ has dimension 1 and degree 24 over this ring, as claimed.

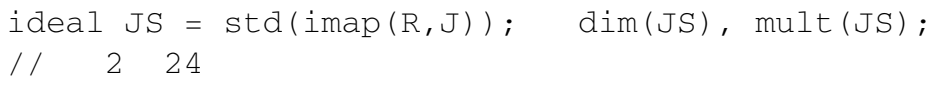

The first ideal in the list $F$ has dimension 0 .

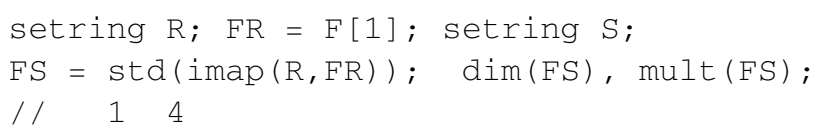

This ideal is a spurious component from the factorization. It is contained in the spurious ideal $E_{2}$.

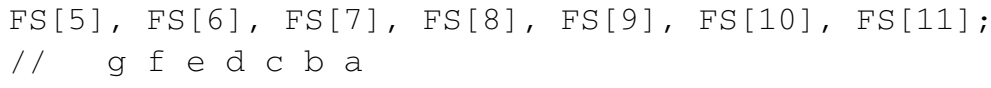

The other six components each have dimension 1 and degree 4 , and each contains a homogeneous quadratic polynomial in the variables $x$ and $y$.

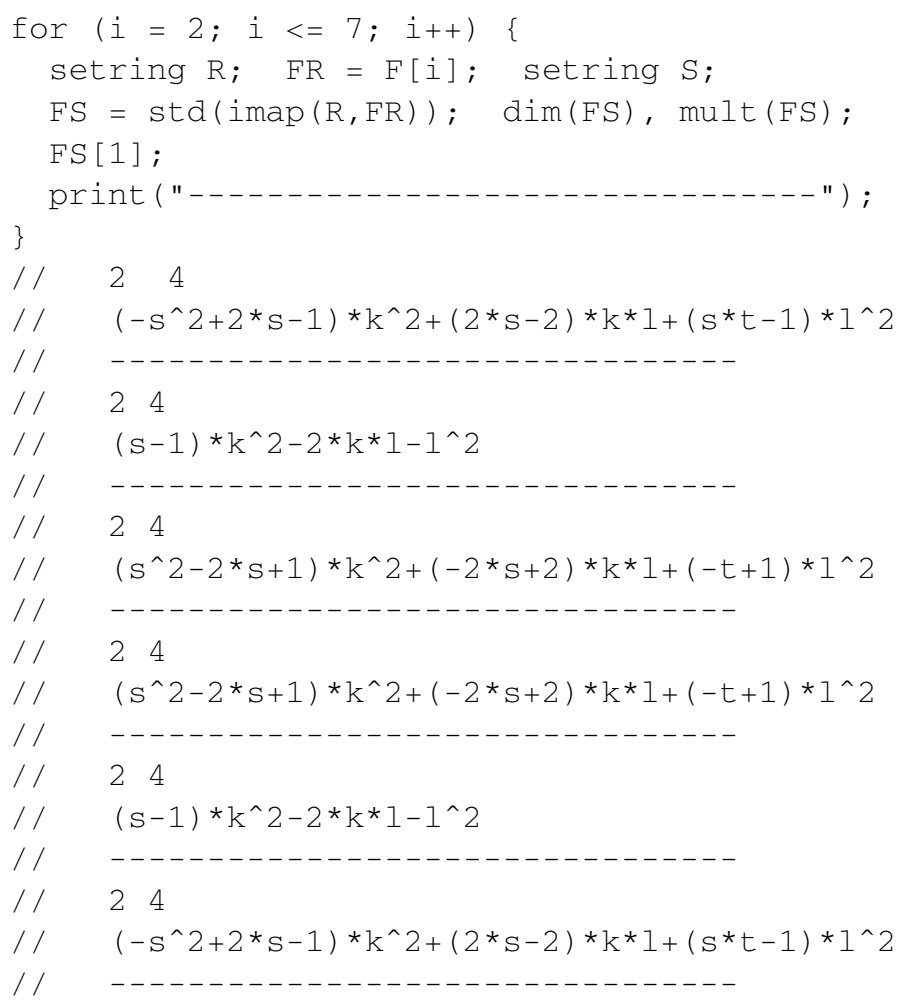


The whole computation takes 7 minutes CPU time on an 800 Mhz Pentium III processor, and 3 minutes of that time are spent on the facstd operation.

Each of these homogeneous quadratic polynomials factors over $\mathbb{Q}(\sqrt{s}, \sqrt{t})$, and induces a factorization of the corresponding ideal. We describe this factorization-which is carried out by hand-in detail for the second component $F_{2}$. We start from the Gröbner basis of the ideal $F_{2}$ computed in the program above,

$$
\begin{gathered}
(s-1) k^{2}-2 k l-l^{2},(s-1) h+(2 t-2) k+(t-1) l, f l-g k, \\
e l-g^{2}, d+f+g, c, 2 b+e, a, \\
(s-1) f k-2 g k-g l,(s-1) f^{2}-2 f g-g^{2}, e k-f g .
\end{gathered}
$$

Over $\mathbb{Q}(\sqrt{s}, \sqrt{t})$, the first polynomial factors into

$$
((\sqrt{s}+1) k+l)((\sqrt{s}-1) k-l) .
$$

We consider the first factor; the second one can be treated similarly. Substituting $l=$ $-(\sqrt{s}+1) k$ into the generator $f l-g k$, that one factors into

$$
-k((\sqrt{s}+1) f+g) .
$$

Since any zero of $F_{2}$ with $k=0$ would imply $a=c=d=f=g=h=k=l=0$ and thus be contained in $\mathcal{V}\left(E_{3}\right)$, we can divide by $k$ and obtain a linear polynomial. Altogether, the first two rows of (A.1) become a set of seven independent linear polynomials and one quadratic polynomial $e l-g^{2}$. For any pair $(s, t)$ satisfying (2.6) they define a plane conic. We leave it to the reader to verify that the three polynomials in the third row are contained in the ideal generated by the first two rows, after the substitution.

In order to show that for none of the parameters $s, t$ satisfying (2.6) the map $\varphi$ vanishes identically on this conic, consider the following point $p=(a, b, c, d, e, d, f, g, h, k, l)$ on it:

$$
\begin{aligned}
& (0,-(\sqrt{s}+1)(s-1), 0,-2 \sqrt{s}(s-1), \\
& 2(\sqrt{s}+1)(s-1),-2(s-1), \\
& 2(\sqrt{s}+1)(s-1), 4(t-1)-2(t-1)(\sqrt{s}+1),-2(s-1), \\
& \left.2(\sqrt{s}+1)(s-1)^{2}\right) .
\end{aligned}
$$

The coefficient of $w^{2} z^{2}$ in $\varphi(C)$ is

$$
-4 s(\sqrt{s}-1)^{2}(\sqrt{s}+1)^{2},
$$

so $\varphi(C)$ does not not vanish identically.

In order to show that for all parameters $s, t$ satisfying (2.6) the 12 conics are distinct, consider the quadratic polynomials in $k$ and $l$ in the SINGULAR output above. In the factorization over $\mathbb{Q}(\sqrt{s}, \sqrt{t})$, the ideal of each of the 12 conics contains a generator which is linear in $k$ and $l$ and independent of $a, \ldots, h$. To show the distinctness of two conics, we distinguish two cases.

If these linear homogeneous polynomials are distinct (over $\mathbb{Q}(s, t)$ ), then it can be checked that for every given pair $(s, t)$ they define subspaces whose restrictions to $(k, l) \neq(0,0)$ are disjoint. 
In the case that the linear homogeneous polynomials coincide, then it can be explicitly checked that both conics are distinct. For example, both $F_{2}$ and $F_{5}$ contain the factor $(\sqrt{s}+1) k+l$ in the first polynomial. As seen above, the corresponding conic of $F_{2}$ is contained in the subspace $a=c=0$. Similarly, the corresponding conic of $F_{5}$ is contained in $e=g=0$. Assuming that the two conics are equal for some pair $(s, t)$, the equations of the ideals can be used to show further that $a=b=c=\cdots=h=0$. However, due to the saturation with the excess component $E_{2}$ this is not possible, and hence the two conics are distinct.

The same calculations for the other components are archived at the web page of this paper, http://www.math.umass.edu/ sottile/pages/212s.html and its mirrors maintained by the other authors.

\section{References}

1. P.K. Agarwal, B. Aronov, and M. Sharir, Computing envelopes in four dimensions with applications, SIAM J. Comput. 26 (1997), 1714-1732.

2. D. Cox, J. Little, and D. O'Shea, Ideals, Varieties, and Algorithms: An Introduction to Computational Algebraic Geometry and Commutative Algebra, 2nd edn., UTM, Springer-Verlag, New York, 1996.

3. D. Eisenbud, Commutative Algebra with a View Towards Algebraic Geometry, GTM, no. 150, SpringerVerlag, New York, 1995.

4. G.-M. Greuel, G. Pfister, and H. Schönemann, Singular 2.0. A computer algebra system for polynomial computations. Centre for Computer Algebra, University of Kaiserslautern, 2001. http://www.singular.uni-kl.de.

5. R. Hartshorne, Algebraic Geometry, GTM, no. 52, Springer-Verlag, New York, 1977.

6. W.V.D. Hodge and D. Pedoe, Methods of Algebraic Geometry, vol. I, Cambridge University Press, Cambridge, 1947.

7. I.G. Macdonald, J. Pach, and T. Theobald, Common tangents to four unit balls in $\mathbb{R}^{3}$, Discrete Comput. Geom. 26 (2001), 1-17.

8. G. Megyesi, Lines tangent to four unit spheres with coplanar centres, Discrete Comput. Geom. 26 (2001), 493-497.

9. H.M. Möller, H. Melenk, and W. Neun, Symbolic solution of large stationary chemical kinetics problems, Impact Comput. Sci. Eng. 1 (1989), 138-167.

10. M. Pellegrini, Ray shooting and lines in spaces, in The CRC Handbook of Discrete and Computational Geometry (J.E. Goodman and J. O’Rourke, eds.), CRC Press, Boca Raton, FL, 1997, pp. 599-614.

11. H. Pottmann and J. Wallner, Computational Line Geometry, Springer-Verlag, Berlin, 2001.

12. J.G. Semple and G.T. Kneebone, Algebraic Curves, Oxford University Press, Oxford, 1959.

13. F. Sottile, From enumerative geometry to solving systems of equations, in Computations in Algebraic Geometry with Macaulay 2 (D. Eisenbud, D. Grayson, M. Stillman, and B. Sturmfels, eds.), ACM, no. 8, Springer-Verlag, Berlin, 2001, pp. 101-129.

14. T. Theobald, An enumerative geometry framework for algorithmic line problems in $\mathbb{R}^{3}$, SIAM J. Comput. 31 (2002), 1212-1228.

15. T. Theobald, Visibility computations: from discrete algorithms to real algebraic geometry, in Algorithmic and Quantitative Real Algebraic Geometry (S. Basu and L. Gonzalez-Vega, eds.), AMS, Providence, RI, 2003.

Received June 5, 2002, and in revised form March 28, 2003. Online publication September 19, 2003. 